\title{
Total Synthesis of Viridiofungins A and B
}

Liselle Atkin, Angus Robertson, Jonathan M. White and Mark A. Rizzacasa.

School of Chemistry, The Bio21 Molecular Science and Biotechnology Institute, The University of Melbourne, Victoria, 3010, Australia.

\section{Supporting Information}

\section{Experimental Section}

\section{General}

S2

Preparation of tosylate 10

Preparation of allyl ester 5

Preparation of hydroxyacid S1

Preparation of $\beta$-lactone 11

Preparation of $\beta$-lactone 3

S5

Preparation of viridiofungin B tri-t-butyl ester 16

Preparation of 4-epi-viridiofungin B tri-t-butyl ester $\mathbf{S 2}$

Preparation of viridiofungin $B(2)$ S8

Preparation of desmethylene analogue (17) S8

Preparation of viridiofungin A tri-t-butyl ester 20 S9

Preparation of viridiofungin A (1) S10

${ }^{1} \mathrm{H}$ and ${ }^{13} \mathrm{C}$ NMR data for natural and synthetic viridiofungin $\mathrm{A}(1)$ S12

${ }^{1} \mathrm{H}$ and ${ }^{13} \mathrm{C}$ NMR data for natural and synthetic viridiofungin $\mathrm{B}(2)$ S13

${ }^{1} \mathrm{H}$ and ${ }^{13} \mathrm{C}$ NMR spectra for all new compounds 


\section{General Experimental.}

All reactions were performed under an inert argon or nitrogen atmosphere in flame-dried or oven- dried $\left(150^{\circ} \mathrm{C}\right)$ round bottom flask fitted with rubber septa using magnetic stirring, and a reflux condenser where appropriate. Hot-blocks on a stirrer hotplate with thermostatic control were used for all reactions that were heated. Anhydrous tetrahydrofuran $(\mathrm{THF})$, diethyl ether $\left(\mathrm{Et}_{2} \mathrm{O}\right)$ and dichloromethane $(\mathrm{DCM})$ were obtained from a solvent dispensing system where solvents were dried by passage through two packed columns of neutral alumina under argon. Dry methanol $(\mathrm{MeOH})$ was distilled from magnesium methoxide or $\mathrm{CaSO}_{4}$ and stored over $4 \AA$ molecular sieves. Dry triethylamine $\left(\mathrm{NEt}_{3}\right)$ and acetonitrile $(\mathrm{MeCN})$ were freshly distilled from calcium hydride under nitrogen atmosphere. Diisopropylamine (DIPA) was distilled from $\mathrm{NaOH}$ under nitrogen or argon atmosphere immediately prior to use. Hexamethylphosphoramide (HMPA) and $\mathrm{N}, \mathrm{N}$ dimethylformamide (DMF) were dried by storage over $4 \AA$ molecule sieves. All other commercial reagents were used as received. Grubbs-Hoveyda catalyst was purchased from Sigma-Aldrich. Brine refers to a saturated aqueous solution of $\mathrm{NaCl}$ and petrol refers to petroleum spirits of the fraction boiling between 40 and $60{ }^{\circ} \mathrm{C}$. Flash chromatography was carried out using silica gel 60. Analytical thin layer chromatography (TLC) was conducted on aluminium backed plates $\left(2 \mathrm{~mm}\right.$ silica gel $\left.60 \mathrm{~F}_{254}\right)$ and chromatograms were visualised under UV light ( $365 \mathrm{~nm}$ ) and with solutions of $20 \% \mathrm{w} / \mathrm{w}$ phosphomolybdic acid in ethanol (PMA). Melting points were obtained in open capillaries and are uncorrected. Optical rotations were recorded in a $10.0 \mathrm{~cm}$ microcell and units are deg. $\mathrm{cm}^{2} \mathrm{~g}^{-1}$. Infrared (IR) spectra were recorded using an attenuated total reflectance (ATR) attachment. Highresolution mass spectra (HRMS) were obtained using electrospray ionisation (ESI) on a quadrupole instrument. Nuclear magnetic resonance (NMR) spectra were recorded at 400 , 500 or $600 \mathrm{MHz}$ in $\mathrm{CDCl}_{3}$ or $d_{4}$-methanol and internally referenced to the residual proton resonance in the solvent ( $\delta 7.26 \mathrm{ppm}$ or $3.31 \mathrm{ppm})$. 


\section{Tosylate 10}

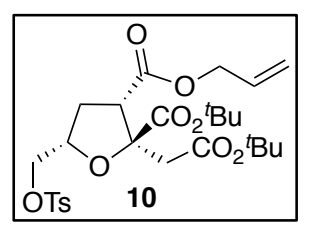

A solution of bicyclic lactone $6^{1}(1.53 \mathrm{~g}, 4.47 \mathrm{mmol})$ and NEt $3(4.0 \mathrm{~mL}$, $28.7 \mathrm{mmol})$ in allyl alcohol $(7.2 \mathrm{~mL})$ was heated at $50^{\circ} \mathrm{C}$ for $21 \mathrm{~h}$. The reaction mixture was cooled to room temperature and diluted with EtOAc $(20 \mathrm{~mL})$. The organic phase was washed with $\mathrm{H}_{2} \mathrm{O}(5 \times 20 \mathrm{~mL})$, brine $(20 \mathrm{~mL})$, dried over $\mathrm{Na}_{2} \mathrm{SO}_{4}$ and concentrated to afford crude hydroxy allyl ester as a yellow oil $(1.40 \mathrm{~g})$, which was used directly in the next step. To a solution of crude alcohol $(1.40 \mathrm{~g})$ in $\mathrm{CH}_{2} \mathrm{Cl}_{2}$ $(21 \mathrm{~mL})$ at $0^{\circ} \mathrm{C}$ was added $\mathrm{TsCl}(1.74 \mathrm{~g}, 9.13 \mathrm{mmol})$, followed by DMAP (91 $\mathrm{mg}, 0.74$ $\mathrm{mmol})$, and then $\mathrm{NEt}_{3}(1.7 \mathrm{~mL}, 12.2 \mathrm{mmol})$. The solution was stirred at room temperature for $21 \mathrm{~h}$ before being diluted with EtOAc $(10 \mathrm{~mL})$ and quenched with $\mathrm{H}_{2} \mathrm{O}(30 \mathrm{~mL})$. The organic phase was washed with $1 \mathrm{M}$ aq. $\mathrm{HCl}(30 \mathrm{~mL})$, followed by a sat. aq. solution of $\mathrm{NaHCO}_{3}(30 \mathrm{~mL}), \mathrm{H}_{2} \mathrm{O}(30 \mathrm{~mL})$, then brine $(30 \mathrm{~mL})$, dried over $\mathrm{Na}_{2} \mathrm{SO}_{4}$ and concentrated under reduced pressure. The crude material was purified by flash chromatography (20\% EtOAc in hexane) to afford tosylate 10 (2.36 g, 95\% from lactone 6) as a colorless oil. $[\alpha]_{D}^{21}=+31.1^{\circ}\left(c 1.47, \mathrm{CH}_{2} \mathrm{Cl}_{2}\right)$; IR $v_{\max }($ film) 2979, 2933, 1733, 1652, 1599, 1456, 1394, 1367, 1257, 1232, 1189, 1176, 1096, 1062, 1020, 983, 887, 840, 815, 791, $665 \mathrm{~cm}^{-1} ;{ }^{1} \mathrm{H}$ NMR (600 MHz, CDCl $) \delta 7.78(\mathrm{~d}, J=8.3 \mathrm{~Hz}, 2 \mathrm{H}, \mathrm{ArH}), 7.32(\mathrm{~d}, J=8.2 \mathrm{~Hz}, 2 \mathrm{H}, \mathrm{ArH}), 5.89$ (ddt, $J=16.9,10.6,6.1 \mathrm{~Hz}, 1 \mathrm{H}, \mathrm{OCH}_{2} \mathrm{CH}=\mathrm{CH}_{2}$ ), $5.32(\mathrm{dd}, J=17.2,1.3 \mathrm{~Hz}, 1 \mathrm{H}$, $\mathrm{OCH}_{2} \mathrm{CH}=\mathrm{CH}_{2}$ ), 5.26 (dd, $J=10.4,0.9 \mathrm{~Hz}, 1 \mathrm{H}, \mathrm{OCH}_{2} \mathrm{CH}=\mathrm{CH}_{2}$ ), 4.60 (dd, $J=13.0,6.0 \mathrm{~Hz}$, $1 \mathrm{H}, \mathrm{TsOCH}_{2}$ ), 4.53 (dd, $\left.J=13.0,6.0 \mathrm{~Hz}, 1 \mathrm{H}, \mathrm{TsOCH}_{2}\right), 4.40\left(\mathrm{~m}, 1 \mathrm{H}, \mathrm{TsOCH}_{2} \mathrm{CH}\right), 4.13$ (d, $\left.J=5.5 \mathrm{~Hz}, 2 \mathrm{H}, \quad \mathrm{OCH}_{2} \mathrm{CH}=\mathrm{CH}_{2}\right), 3.35\left(\mathrm{dd}, J=8.2,6.5 \mathrm{~Hz}, 1 \mathrm{H}, \quad \mathrm{CHCO}_{2} \mathrm{CH}_{2} \mathrm{CH}=\mathrm{CH}_{2}\right.$ ), 2.77 (d, $J=16.8 \mathrm{~Hz}, 1 \mathrm{H}, \mathrm{CH}_{2} \mathrm{CO}_{2} \mathrm{tBu}$ ), 2.64 (d, $J=16.8 \mathrm{~Hz}, 1 \mathrm{H}, \mathrm{CH}_{2} \mathrm{CO}_{2} \mathrm{tBu}$ ), 2.43 (s, 3H, $\mathrm{CH}_{3} \mathrm{Ar}$ ), 2.31 (dt, $J=13.0,7.8 \mathrm{~Hz}, 1 \mathrm{H}, \mathrm{CH}_{2} \mathrm{CHCO}_{2} \mathrm{CH}_{2} \mathrm{CH}=\mathrm{CH}_{2}$ ), 2.10 (dt, $J=13.3,6.7$ $\mathrm{Hz}, 1 \mathrm{H}, \mathrm{CH}_{2} \mathrm{CHCO}_{2} \mathrm{CH}_{2} \mathrm{CH}=\mathrm{CH}_{2}$ ), 1.45 (s, 9H, $\mathrm{CO}_{2}{ }^{t} \mathrm{Bu}$ ), 1.40 (s, 9H, $\mathrm{CO}_{2}{ }^{t} \mathrm{Bu}$ ) ppm; ${ }^{13} \mathrm{C}$ NMR $\left(100 \mathrm{MHz}, \mathrm{CDCl}_{3}\right) \delta 171.3,170.6,168.8,144.9,132.9,131.7,129.9,128.2,119.4$, 85.7, 82.3, 81.3, 76.8, 70.7, 66.6, 50.0, 40.4, 31.9, 28.1, 27.9, 24.0, 21.8 ppm; HRMS (ESI) $m / z$ : $[\mathrm{M}+\mathrm{Na}]^{+}$Calcd for $\mathrm{C}_{27} \mathrm{H}_{38} \mathrm{O}_{10} \mathrm{SNa} 577.2083$; Found 577.2078.

\section{Allyl ester 5}

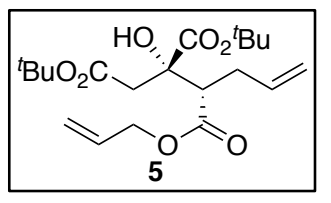

A solution of tosylate $10(960 \mathrm{mg}, 1.73 \mathrm{mmol})$ and $\mathrm{Nal}(5.19 \mathrm{~g}, 34.6$ $\mathrm{mmol}$ ) in acetone $(18 \mathrm{~mL})$ was stirred at $70^{\circ} \mathrm{C}$ for $19 \mathrm{~h}$ before being cooled to room temperature, filtered through Celite and concentrated.

(1) Chen, Z.; Robertson, A.; White, J. M.; Rizzacasa, M. A. Org. Lett. 2019, 21, 9663-9666. 
To a solution of the crude iodide in aqueous EtOH $(25 \mathrm{~mL}, 95 \% \mathrm{v} / \mathrm{v})$ was added zinc powder $(790 \mathrm{mg}, 12.1 \mathrm{mmol}$, freshly activated by stirring with $10 \%$ aq. $\mathrm{HCl}$ solution for 30 mins then filtering and washing with $\mathrm{H}_{2} \mathrm{O}$, EtOH and $\left.\mathrm{Et}_{2} \mathrm{O}\right)$ and solid $\mathrm{NH}_{4} \mathrm{Cl}(270 \mathrm{mg}, 5.05$ $\mathrm{mmol}$ ) and the reaction mixture was stirred at room temperature for $17 \mathrm{~h}$ then concentrated. The crude residue was dissolved in EtOAc $(50 \mathrm{~mL})$ and then filtered through a plug of Celite. The organic phase was then washed with brine $(50 \mathrm{~mL})$, dried over $\mathrm{Na}_{2} \mathrm{SO}_{4}$ and the residue was purified by flash chromatography (10\% EtOAc in hexane) to afford alkene allyl ester 5 (587 mg, $88 \%)$ as a yellow oil. $[\alpha]_{D}^{19}=+16.8^{\circ}\left(c 0.36, \mathrm{CH}_{2} \mathrm{Cl}_{2}\right)$; IR $v_{\max }$ (film) 3489, 3085, 2980, 2934, 1733, 1644, 1478, 1457, 1394, 1369, 1290, 1250, 1150, 1117, 1053, 987, 919, 868, 845, 824, 753.6, 725, $702 \mathrm{~cm}^{-1} ;{ }^{1} \mathrm{H}$ NMR (600 MHz, $\mathrm{CDCl}_{3}$ ) $\delta 5.91$ (ddt, $J=16.9,11.0,5.7 \mathrm{~Hz}, 1 \mathrm{H}, \mathrm{CH}=\mathrm{CH}_{2}$ ), 5.69 (ddt, $J=17.0,10.1,6.9 \mathrm{~Hz}$, $\left.1 \mathrm{H}, \mathrm{CH}=\mathrm{CH}_{2}\right), 5.34\left(\mathrm{dd}, J=17.2,1.3 \mathrm{~Hz}, 1 \mathrm{H}, \mathrm{CH}=\mathrm{CH}_{2}\right), 5.24(\mathrm{dd}, J=10.4,1.1 \mathrm{~Hz}, 1 \mathrm{H}$, $\mathrm{CH}=\mathrm{CH}_{2}$ ), 5.06 (dd, $\left.J=17.1,1.3 \mathrm{~Hz}, 1 \mathrm{H}, \mathrm{CH}=\mathrm{CH}_{2}\right), 5.01\left(\mathrm{~d}, J=10.2 \mathrm{~Hz}, 1 \mathrm{H}, \mathrm{CH}=\mathrm{CH}_{2}\right.$ ), 4.64 (dd, $J=13.2,5.8 \mathrm{~Hz}, 1 \mathrm{H}, \mathrm{OCH}_{2} \mathrm{CH}=\mathrm{CH}_{2}$ ), 4.56 (dd, $J=13.2,5.9 \mathrm{~Hz}, \mathrm{OCH}_{2} \mathrm{CH}=\mathrm{CH}_{2}$ ), $3.96(\mathrm{~s}, 1 \mathrm{H}, \mathrm{OH}), 2.99\left(\mathrm{~d}, J=16.6 \mathrm{~Hz}, 1 \mathrm{H}, \mathrm{CH}_{2} \mathrm{CO}_{2}{ }^{\mathrm{B}} \mathrm{Bu}\right), 2.75(\mathrm{dd}, J=11.8,3.2 \mathrm{~Hz}, 1 \mathrm{H}$, $\left.\mathrm{CHCO}_{2} \mathrm{CH}_{2} \mathrm{CH}=\mathrm{CH}_{2}\right), 2.64\left(\mathrm{~d}, J=16.6 \mathrm{~Hz}, 1 \mathrm{H}, \mathrm{CH}_{2} \mathrm{CO}_{2} \mathrm{~B} \mathrm{Bu}\right), 2.55(\mathrm{td}, J=13.0,7.5 \mathrm{~Hz}, 1 \mathrm{H}$, $\left.\mathrm{CHCH}_{2} \mathrm{CH}=\mathrm{CH}_{2}\right), 2.27-2.31\left(\mathrm{~m}, 1 \mathrm{H}, \mathrm{CHCH}_{2} \mathrm{CH}=\mathrm{CH}_{2}\right), 1.49\left(\mathrm{~s}, 9 \mathrm{H},{ }^{t} \mathrm{Bu}\right), 1.43\left(\mathrm{~s}, 9 \mathrm{H},{ }^{t} \mathrm{Bu}\right)$ ppm; ${ }^{13} \mathrm{C}$ NMR $\left(125.5 \mathrm{MHz}, \mathrm{CDCl}_{3}\right) \delta 172.7,171.4,169.9,134.9,132.0,118.8,117.4$, 83.4, 81.6, 75.2, 65.6, 53.7, 42.0, 31.7, 28.2, 27.99, 27.97 ppm; HRMS (ESI) m/z: Calcd for $\mathrm{C}_{20} \mathrm{H}_{32} \mathrm{O}_{7} \mathrm{Na}[\mathrm{M}+\mathrm{Na}]^{+}$407.2046; Found 407.2038.

\section{Hydroxyacid S1}

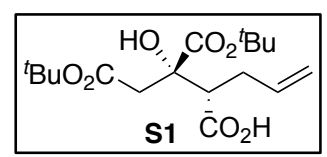

To a solution of the allyl ester $5(300 \mathrm{mg}, 0.780 \mathrm{mmol})$ in EtOH $(7.5$ $\mathrm{mL}) . \mathrm{H}_{2} \mathrm{O}(0.9 \mathrm{~mL})$ was added Wilkinson's catalyst $(72 \mathrm{mg}, 0.078 \mathrm{mmol}$, $10 \mathrm{~mol} \%$ ) and the reaction mixture was heated to reflux for $19 \mathrm{~h}$, cooled to room temperature and then concentrated under reduced pressure. The crude product was then purified by flash chromatography (30\% EtOAc in hexane with $1 \% \mathrm{AcOH})$ to give the hydroxyacid $\mathbf{S 1}(256 \mathrm{mg}, 95 \%)$ as a yellow oil. $[\alpha]_{D}^{19}=-2.1^{\circ}\left(c 1.33, \mathrm{CH}_{2} \mathrm{Cl}_{2}\right)$; IR $v_{\max }($ film) 2978, 2934, 2869, 1734, 1457, 1393, 1368, 1291, 1250, 1150, 1116, 996, 916, 869, 843, 794, 753, 733, 668, 641, 529, $438 \mathrm{~cm}^{-1} ;{ }^{1} \mathrm{H}$ NMR $\left(600 \mathrm{MHz}, \mathrm{CDCl}_{3}\right) \delta 5.74$ (dddd, J = 17.1, 10.1, 7.2, $6.8 \mathrm{~Hz}, 1 \mathrm{H}, \mathrm{CH}_{2}=\mathrm{CH}$ ), 5.11 (dd, $J=17.0,1.4 \mathrm{~Hz}, 1 \mathrm{H}, \mathrm{CH}_{2}=\mathrm{CH}$ ), 5.07 (d, J = 10.2 $\mathrm{Hz}, 1 \mathrm{H}, \mathrm{CH}_{2}=\mathrm{CH}$ ), $2.96\left(\mathrm{~d}, J=16.7 \mathrm{~Hz}, 1 \mathrm{H}, \mathrm{CH}_{2} \mathrm{CO}_{2} \mathrm{Bu}\right), 2.74$ (dd, $J=11.5,3.5 \mathrm{~Hz}, 1 \mathrm{H}$, $\mathrm{CH}_{2} \mathrm{CHCO}_{2} \mathrm{H}$ ), 2.71 (d, $J=16.7 \mathrm{~Hz}, 1 \mathrm{H}, \mathrm{CH}_{2} \mathrm{CO}_{2}{ }^{\mathrm{t}} \mathrm{Bu}$ ), 2.49 (td, $J=12.8,7.7 \mathrm{~Hz}, 1 \mathrm{H}$, 
$\left.\mathrm{CH}_{2} \mathrm{CHCO}_{2} \mathrm{H}\right), 2.23-2.26\left(\mathrm{~m}, 1 \mathrm{H}, \mathrm{CH}_{2} \mathrm{CHCO}_{2} \mathrm{H}\right), 1.50\left(\mathrm{~s}, 9 \mathrm{H}, \mathrm{CO}_{2}{ }^{t} \mathrm{Bu}\right), 1.44(\mathrm{~s}, 9 \mathrm{H}$, $\left.\mathrm{CO}_{2}{ }^{\mathrm{Bu}} \mathrm{B}\right) \mathrm{ppm} ;{ }^{13} \mathrm{C} \mathrm{NMR}\left(125.5 \mathrm{MHz}, \mathrm{CDCl}_{3}\right) \delta 172.2,172.1,169.7,134.3,134.3,117.9$, 117.9, 84.0, 84.0, 82.0, 81.9, 75.0, 53.4, 42.2, 31.4, 28.2, 28.0 ppm; HRMS (ESI) $\mathrm{m} / \mathrm{z}$ : Calcd for $\mathrm{C}_{17} \mathrm{H}_{29} \mathrm{O}_{7}[\mathrm{M}+\mathrm{H}]^{+} 367.1733$; Found 367.1726 .

\section{$\beta$-Lactone 11}

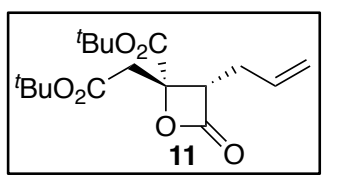

A solution of the acid $\mathbf{S 1}(2.0 \mathrm{~g}, 5.81 \mathrm{mmol})$, DCC $(2.72 \mathrm{~g}, 13.2 \mathrm{mmol})$ and DMAP (79 mg, $0.647 \mathrm{mmol})$ in $\mathrm{CH}_{2} \mathrm{Cl}_{2}(19.4 \mathrm{~mL}$ ) was stirred for 3.5 $\mathrm{h}$ at room temperature then filtered through Celite and concentrated.

The crude material was purified by flash chromatography (5\% EtOAc in hexane) to afford $\beta$-lactone $11(1.51 \mathrm{~g}, 80 \%)$ as a colorless low-melting solid. $[\alpha]_{D}^{20}=-31.0^{\circ}\left(c 0.82, \mathrm{CH}_{2} \mathrm{Cl}_{2}\right)$; IR $v_{\max }$ (film) 2980, 2933, 1838, 1734, 1646, 1478, 1457, 1395, 1369, 1319, 1285, 1251 , 1224, 1151, 982, 922, 855, $762 \mathrm{~cm}^{-1} ;{ }^{1} \mathrm{H}$ NMR $\left(500 \mathrm{MHz}, \mathrm{CDCl}_{3}\right) \delta 5.82$ (ddt, $J=17.0$, 10.4, $6.5 \mathrm{~Hz}, 1 \mathrm{H}, \mathrm{CH}_{2}=\mathrm{CH}$ ), $5.12-5.17\left(\mathrm{~m}, 2 \mathrm{H}, \mathrm{CH}_{2}=\mathrm{CH}\right.$ ), $3.73(\mathrm{dd}, J=8.5,7.8 \mathrm{~Hz}, 1 \mathrm{H}$, $\mathrm{CH}_{2} \mathrm{CHC}(\mathrm{O})$ ), 3.19 (dd, $J=16.8 \mathrm{~Hz}, 1 \mathrm{H}, \mathrm{CH}_{2} \mathrm{CO}_{2} \mathrm{Bu}$ ), $2.89(\mathrm{~d}, J=16.8 \mathrm{~Hz}, 1 \mathrm{H}$, $\mathrm{CH}_{2} \mathrm{CO}_{2}{ }^{2} \mathrm{Bu}$ ), 2.53-2.59 (m, $\left.1 \mathrm{H}, \mathrm{CH}_{2} \mathrm{CH}(\mathrm{O})\right)$, 2.41-2.49 (m, $\left.1 \mathrm{H}, \mathrm{CH}_{2} \mathrm{CH}(\mathrm{O})\right), 1.52(\mathrm{~s}, 9 \mathrm{H}$, $\left.\mathrm{CO}_{2}{ }^{\mathrm{B} u}\right), 1.46$ (s, 9H, $\mathrm{CO}_{2}{ }^{\mathrm{t}} \mathrm{Bu}$ ) ppm; ${ }^{13} \mathrm{C} \mathrm{NMR}\left(100 \mathrm{MHz}, \mathrm{CDCl}_{3}\right) \delta$ 168.6, 167.4, 167.0, 132.9, 118.0, 84.4, 82.4, 77.8, 58.8, 42.1, 29.4, 28.1, 28.1 ppm; HRMS (ESI) m/z: Calcd for $\mathrm{C}_{17} \mathrm{H}_{26} \mathrm{O} 6 \mathrm{Na}[\mathrm{M}+\mathrm{Na}]^{+} 349.1627$; Found 349.1617.

\section{$\beta$-Lactone 3}

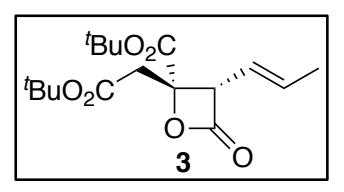

To a solution of the $\beta$-lactone 11 (56 $\mathrm{mg}, 0.172 \mathrm{mmol}$ ) in $\mathrm{MeOH}(2 \mathrm{~mL})$ was added Grubbs generation II catalyst (14.9 $\mathrm{mg}, 0.0176 \mathrm{mmol}$ ) and the reaction mixture was heated at reflux for $20 \mathrm{~h}$ before being concentrated. The crude product was purified by flash chromatography $(10 \%$ EtOAc in hexane) to afford isomerized $\beta$-lactone $3(45.6 \mathrm{mg}, 81 \%, 6.7: 1 \mathrm{E} / Z$ ) as colorless needles. m.p. $107.9-111.3^{\circ} \mathrm{C} ;[\alpha]_{D}^{20}=-27.3^{\circ}\left(c 1.12, \mathrm{CH}_{2} \mathrm{Cl}_{2}\right)$; IR $v_{\max }$ (film) 2979, 2934, 1836, 1736, 1731, 1477, 1455, 1394, 1368, 1317, 1285, 1226, 1142, 1078, 1038, 973, 944, 897, 843, 822, 759, 715, 697, 590, 551, 465, $438 \mathrm{~cm}^{-1}$; Data for E-isomer: ${ }^{1} \mathrm{H}$ NMR $(500 \mathrm{MHz}$, $\mathrm{CDCl}_{3}$ ) $\delta 5.87$ (ddd, $J=15.4,6.6,1.2 \mathrm{~Hz}, 1 \mathrm{H}, \mathrm{CH}=\mathrm{CHCH}_{3}$ ), 5.42 (ddq, $J=15.4,7.7,1.7$, $\left.1 \mathrm{H}, \mathrm{CH}=\mathrm{CHCH}_{3}\right), 4.29(\mathrm{dt}, J=7.7,1.0 \mathrm{~Hz}, 1 \mathrm{H}, \mathrm{CHC}(\mathrm{O})), 3.19(\mathrm{~d}, J=16.7 \mathrm{~Hz}, 1 \mathrm{H}$, $\mathrm{CH}_{2} \mathrm{CO}_{2}{ }^{t} \mathrm{Bu}$ ), 2.89 (d, $J=16.7 \mathrm{~Hz}, 1 \mathrm{H}, \mathrm{CH}_{2} \mathrm{CO}_{2}{ }^{t} \mathrm{Bu}$ ), 1.72 (ddd, $J=6.6,1.7,0.9 \mathrm{~Hz}, 3 \mathrm{H}$, $\left.\mathrm{CH}_{3} \mathrm{CH}=\mathrm{CH}\right), 1.48\left(\mathrm{~s}, 9 \mathrm{H}, \mathrm{CO}_{2}{ }^{t} \mathrm{Bu}\right), 1.46\left(\mathrm{~s}, 9 \mathrm{H}, \mathrm{CO}_{2}{ }^{\mathrm{t}} \mathrm{Bu}\right) \mathrm{ppm} ;{ }^{13} \mathrm{C} \mathrm{NMR}(125 \mathrm{MHz}$, 
$\left.\mathrm{CDCl}_{3}\right) \delta$ 167.6, 167.3, 166.6, 134.4, 118.3, 83.7, 82.2, 78.5, 62.1, 41.4, 28.03, 27.99, 18.1 ppm; HRMS (ESI) $\mathrm{m} / \mathrm{z}$ : Calcd for $\mathrm{C}_{17} \mathrm{H}_{26} \mathrm{O}_{6} \mathrm{Na}[\mathrm{M}+\mathrm{Na}]^{+} 349.1627$; Found 349.1646. Data for Z-isomer: ${ }^{1} \mathrm{H}$ NMR $\left(500 \mathrm{MHz}, \mathrm{CDCl}_{3}\right): \delta$ 5.83-5.90 (m, overlap with $E$ isomer, $\mathrm{CH}=\mathrm{CHCH}_{3}$ ), 5.47 (ddd, $J=10.7,8.8,1.8,1 \mathrm{H}, \mathrm{CH}=\mathrm{CHCH}_{3}$ ), 4.61 (dd, $J=8.8,0.9 \mathrm{~Hz}, 1 \mathrm{H}$, $\mathrm{CHC}(\mathrm{O})), 3.26\left(\mathrm{~d}, J=16.8 \mathrm{~Hz}, 1 \mathrm{H}, \mathrm{CH}_{2} \mathrm{CO}_{2}{ }^{2} \mathrm{Bu}\right), 2.93\left(\mathrm{~d}, J=16.8 \mathrm{~Hz}, 1 \mathrm{H}, \mathrm{CH}_{2} \mathrm{CO}_{2}{ }^{t} \mathrm{Bu}\right.$ ), $1.69\left(\mathrm{dd}, J=6.9,1.8 \mathrm{~Hz}, 3 \mathrm{H}, \mathrm{CH}_{3} \mathrm{CH}=\mathrm{CH}\right), 1.49\left(\mathrm{~s}, 9 \mathrm{H}, \mathrm{CO}_{2}{ }^{\mathrm{t}} \mathrm{Bu}\right), 1.46\left(\mathrm{~s}, 9 \mathrm{H}, \mathrm{CO}_{2}{ }^{\mathrm{t}} \mathrm{Bu}\right)$ ppm.

\section{Viridiofungin $B$ tri-t-butyl ester 16}

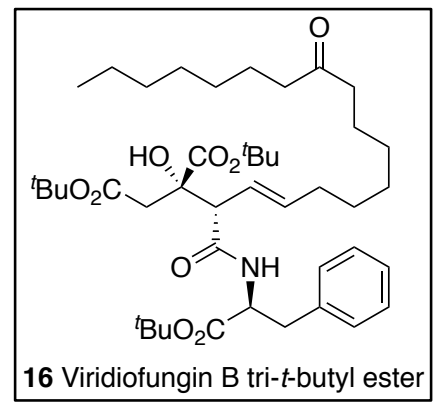

A solution of $\beta$-lactone 3 (86 mg, $0.264 \mathrm{mmol})$, alkene $4^{2}(189 \mathrm{mg}$, $0.793 \mathrm{mmol}$ ), 2,6-dichloro-1,4-benzoquinone (5 mg, $0.028 \mathrm{mmol}$ ) and Grubbs-Hoveyda catalyst (12) (33 mg, $0.053 \mathrm{mmol}$ ) in $\mathrm{CH}_{2} \mathrm{Cl}_{2}$ (3.4 mL) was refluxed for $18 \mathrm{~h}$ then concentrated. The crude material was quickly purified by flash chromatography (5-10\% EtOAc in hexane) to afford cross metathesis product $13(108 \mathrm{mg}$, contaminated with the homodimer derived from alkene 4). This was dissolved in THF (3.8 $\mathrm{mL}$ ) and L-phenylalanine-tert-butyl ester hydrochloride (15) (203 mg, $0.789 \mathrm{mmol}, 3 \mathrm{eq}$ ) and sodium-2-ethylhexanoate (14) (87 mg, $0.523 \mathrm{mmol}, 2 \mathrm{eq}$ ) were added and the reaction was heated under reflux for $18 \mathrm{~h}$. The solution was cooled to room temperature before ethyl acetate $(15 \mathrm{~mL})$ and water $(15 \mathrm{~mL})$ were added. The aqueous layer was extracted into ethyl acetate $(3 \times 15 \mathrm{~mL})$, and the combined organic layers were washed with water $(15 \mathrm{~mL})$ and then brine $(15 \mathrm{~mL})$ before being concentrated. The crude material was purified by flash chromatography (10-15 \% ethyl acetate in hexane) to afford pure viridiofungin $B$ tri- $t$-butyl ester (16) $(113.8 \mathrm{mg}, 58 \%$ from 3$)$ as a colorless oil. $[\alpha]_{D}^{22}=-22.3^{\circ}$ (c 0.55, $\mathrm{CH}_{2} \mathrm{Cl}_{2}$ ); IR $v_{\max }$ (film) 3370, 2978, 2930, 2857, 1733, 1678, 1524, 1456, 1393, $1368,1253,1154,971,848,740,701 \mathrm{~cm}^{-1} ;{ }^{1} \mathrm{H}$ NMR $\left(500 \mathrm{MHz}, \mathrm{CDCl}_{3}\right) \delta$ 7.25-7.27 (m, 2H, ArH), 7.20 (t, $J=7.20 \mathrm{~Hz}, 3 \mathrm{H}, \operatorname{ArH}), 7.02(\mathrm{~d}, J=8.0 \mathrm{~Hz}, 1 \mathrm{H}, \mathrm{NH}), 5.60-5.66(\mathrm{~m}, 1 \mathrm{H}$, $\mathrm{CH}=\mathrm{CHCHC}(\mathrm{O})), 5.45(\mathrm{dd}, J=15.2,9.6 \mathrm{~Hz}, 1 \mathrm{H}, \mathrm{CH}=\mathrm{CHCHC}(\mathrm{O})), 4.71(\mathrm{q}, J=6.8 \mathrm{~Hz}, 1 \mathrm{H}$, CHNH), 4.19 (s, $1 \mathrm{H}, \mathrm{OH}$ ), 3.14 (dd, $J=13.9,5.7 \mathrm{~Hz}, 1 \mathrm{H}, \mathrm{CH}_{2} \mathrm{CHNH}$ ), 3.05 (dd, $J=13.8$, $8.1 \mathrm{~Hz}, 2 \mathrm{H}, \quad \mathrm{CH}_{2} \mathrm{CHNH}$ and $\mathrm{CH}=\mathrm{CHCHC}(\mathrm{O})$ overlapping), $2.69(\mathrm{~d}, J=16.7 \mathrm{~Hz}, 1 \mathrm{H}$, $\mathrm{CH}_{2} \mathrm{CO}_{2}{ }^{\mathrm{B}} \mathrm{Bu}$ ), 2.45 (d, $J=16.7 \mathrm{~Hz}, 1 \mathrm{H}, \mathrm{CH}_{2} \mathrm{CO}_{2}{ }^{\mathrm{B} u} \mathrm{~B}$ ), 2.37 (td, $J=7.3,4.4 \mathrm{~Hz}, 4 \mathrm{H}$, $\left.\mathrm{CH}_{2} \mathrm{C}(\mathrm{O}) \mathrm{CH}_{2}\right), 1.94-1.99\left(\mathrm{~m}, 2 \mathrm{H}, \mathrm{CH}_{2} \mathrm{CH}=\mathrm{CH}\right), 1.50-1.58\left(\mathrm{~m}, 4 \mathrm{H}, \mathrm{CH}_{2}\right), 1.43(\mathrm{~s}, 9 \mathrm{H}$,

(2) Goldup, S. M.; Pilkington, C. J.; White, A. J. P.; Burton, A.; Barrett, A. G. M., J. Org. Chem. 2006, 71, 6185-6191. 
$\left.\mathrm{CO}_{2}{ }^{\mathrm{t}} \mathrm{Bu}\right), 1.42\left(\mathrm{~s}, 9 \mathrm{H}, \mathrm{CO}_{2}{ }^{\mathrm{t}} \mathrm{Bu}\right), 1.39\left(\mathrm{~s}, 9 \mathrm{H}, \mathrm{CO}_{2}{ }^{\mathrm{t}} \mathrm{Bu}\right), 1.27-1.30\left(\mathrm{~m}, 14 \mathrm{H}, \mathrm{CH}_{2}\right), 0.88(\mathrm{t}, J$ $\left.=6.8 \mathrm{~Hz}, 3 \mathrm{H}, \mathrm{CH}_{3}\right) \mathrm{ppm} ;{ }^{13} \mathrm{C} \mathrm{NMR}\left(125.5 \mathrm{MHz}, \mathrm{CDCl}_{3}\right) \delta 211.8,172.0,170.5,170.3$, 169.6, 136.9, 136.6, 129.7, 128.5, 127.0, 122.6, 83.1, 82.2, 81.4, 75.6, 58.4, 53.9, 43.0, 42.9, 42.4, 38.2, 32.7, 31.8, 29.4, 29.2, 29.1, 29.0, 28.2, 28.09, 28.08, 24.0, 23.9, 22.8, $14.2 \mathrm{ppm}$; HRMS (ESI) m/z: Calcd for $\mathrm{C}_{43} \mathrm{H}_{70} \mathrm{NO}_{9}[\mathrm{M}+\mathrm{H}]^{+} 744.5051$; Found 744.5050 .

\section{4-Epi-viridiofungin B tri-t-butyl ester S2}

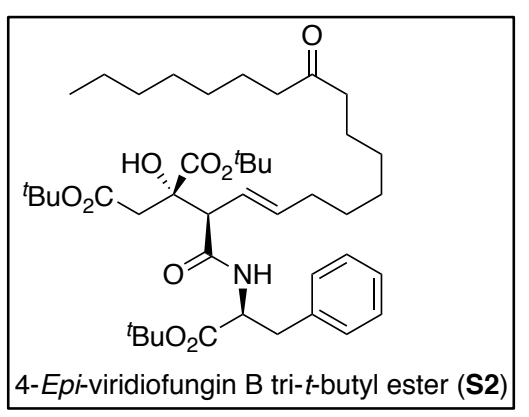

A solution of $\beta$-lactone 3 (50 $\mathrm{mg}, 0.153 \mathrm{mmol})$, alkene 4 (110 $\mathrm{mg}, 0.461 \mathrm{mmol}$ ) and Grubbs-Hoveyda catalyst (12) (19 $\mathrm{mg}, 0.031 \mathrm{mmol})$ in $\mathrm{CH}_{2} \mathrm{Cl}_{2}(2 \mathrm{~mL})$ was heated under reflux for $18.5 \mathrm{~h}$ then concentrated. The crude material was quickly purified by flash chromatography (5\% EtOAc in hexane) to afford cross metathesis product $13(28 \mathrm{mg}$, contaminated with the alkene 4 homodimer and a similar byproduct). A solution of the $\beta$-lactone 13 mixture (28 mg), L-phenylalanine-tert-butyl ester hydrochloride (15) (14 mg, $0.054 \mathrm{mmol}$ ) and sodium-2-ethylhexanoate (14) (14 mg, $0.084 \mathrm{mmol})$ in THF (1.4 mL) was stirred and heated under reflux for $19 \mathrm{~h}$. The solution was cooled to room temperature before ethyl acetate $(10 \mathrm{~mL})$ and water $(10 \mathrm{~mL})$ were added. The aqueous layer was further extracted with ethyl acetate $(3 \times 10 \mathrm{~mL})$, and the combined organic layers were washed with water $(10 \mathrm{~mL})$ and then brine $(10 \mathrm{~mL})$ before being concentrated. The crude material was purified by flash chromatography ( $15 \%$ ethyl acetate in hexane) and then HPLC (5 micron silica column $250 \times 10 \mathrm{~mm}, 20 \%$ ethyl acetate in hexane) to afford viridiofungin B tri-t-butyl ester (16) contaminated with the desmethylene compound $(2.1 \mathrm{mg}, 2 \%$ from 3 ) and 4 -epiviridiofungin B tri-t-butyl ester (S2) $\left(5.0 \mathrm{mg}, 4 \%\right.$ from 3) as a colourless oil. $[\alpha]_{D}^{21}=+49.4^{\circ}$ (c 0.15, $\mathrm{CH}_{2} \mathrm{Cl}_{2}$ ); IR $v_{\max }$ (film) 3329, 2929, 2857, 2166, 2014, 1736, 1644, 1532, 1368, 1248, 1157, 849, $701 \mathrm{~cm}^{-1}$; ${ }^{1} \mathrm{H}$ NMR $\left(500 \mathrm{MHz}, \mathrm{CDCl}_{3}\right) \delta 7.21(\mathrm{dt}, J=13.1,6.8 \mathrm{~Hz}, 3 \mathrm{H}$, ArH), $7.15(\mathrm{~m}, 2 \mathrm{H}, \operatorname{ArH}), 6.72(\mathrm{~d}, J=7.6 \mathrm{~Hz}, 1 \mathrm{H}, \mathrm{NH}), 5.66(\mathrm{dt}, J=15.2,6.7 \mathrm{~Hz}, 1 \mathrm{H}$, $\mathrm{CH}=\mathrm{CHCHC}(\mathrm{O})$ ), $5.48(\mathrm{dd}, J=15.3,10.0 \mathrm{~Hz}, 1 \mathrm{H}, \mathrm{CH}=\mathrm{CHCHC}(\mathrm{O})$ ), 4.67 (dt, $J=7.5,5.9$ $\mathrm{Hz}, 1 \mathrm{H}, \mathrm{CHNH}$ ), 4.45 (s, 1H, OH), $3.18(\mathrm{~d}, J=9.9 \mathrm{~Hz}, 1 \mathrm{H}, \mathrm{CH}=\mathrm{CHCHC}(\mathrm{O})), 3.05$ (dd, $J=$ $5.81,1.41 \mathrm{~Hz}, 2 \mathrm{H}, \mathrm{CH}_{2} \mathrm{CHNH}$ ), $2.61\left(\mathrm{~d}, J=16.1 \mathrm{~Hz}, 1 \mathrm{H}, \mathrm{CH}_{2} \mathrm{CO}_{2}{ }^{\mathrm{B} u} \mathrm{~B}\right), 2.57(\mathrm{~d}, J=16.1$ $\mathrm{Hz}, 1 \mathrm{H}, \mathrm{CH}_{2} \mathrm{CO}_{2} \mathrm{Bu}$ ), 2.37 (t, $\left.J=7.5 \mathrm{~Hz}, 4 \mathrm{H}, \mathrm{CH}_{2} \mathrm{C}(\mathrm{O}) \mathrm{CH}_{2}\right), 2.05$ (q, $J=7.1 \mathrm{~Hz}, 2 \mathrm{H}$, $\mathrm{CH}_{2} \mathrm{CH}=\mathrm{CH}$ ), $1.55\left(\mathrm{t}, J=7.1 \mathrm{~Hz}, 4 \mathrm{H}, \mathrm{CH}_{2}\right), 1.45\left(\mathrm{~s}, 9 \mathrm{H}, \mathrm{CO}_{2}{ }^{\mathrm{B} u}\right), 1.42\left(\mathrm{~s}, 9 \mathrm{H}, \mathrm{CO}_{2}{ }^{\mathrm{B} u}\right)$, $1.38\left(\mathrm{~s}, 9 \mathrm{H}, \mathrm{CO}_{2}{ }^{\mathrm{t}} \mathrm{Bu}\right), 1.26-1.29\left(\mathrm{~m}, 14 \mathrm{H}, \mathrm{CH}_{2}\right), 0.87$ (t, $\left.J=7.0 \mathrm{~Hz}, 3 \mathrm{H}, \mathrm{CH}_{3}\right) \mathrm{ppm} ;{ }^{13} \mathrm{C}$ 
NMR (125 MHz, $\left.\mathrm{CDCl}_{3}\right) \delta 211.8,172.4,170.21,170.15,170.06,138.1,136.4,129.9$, 128.3, $126.9123 .1,82.9,82.3,81.6,75.7,57.6,53.72,53.69,43.0,42.9,42.5,38.3$, 32.8, 31.8, 29.4, 29.2, 29.0, 28.2, 28.1, 28.0, 24.04, 23.95, 22.8, 14.2 ppm; HRMS (ESI) m/z: Calcd for $\mathrm{C}_{43} \mathrm{H}_{69} \mathrm{NO}_{9} \mathrm{Na}[\mathrm{M}+\mathrm{Na}]^{+}$766.4870; Found 766.4839.

\section{Viridiofungin B (2)}

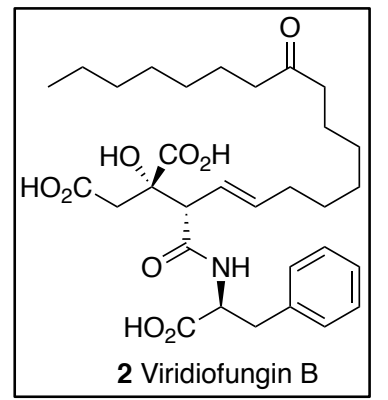

Viridiofungin B tri-t-butyl ester (16) (11 $\mathrm{mg}, 0.015 \mathrm{mmol})$ was dissolved in formic acid $(2 \mathrm{~mL})$ and the reaction mixture was stirred at room temperature for $3 \mathrm{~h}$ before being concentrated to afford viridiofungin $\mathrm{B}(2)$ as an amorphous solid (8.5 $\mathrm{mg}, 98 \%) .[\alpha]_{D}^{23}=-$ $21.3^{\circ}$ (c 0.30, $\mathrm{CH}_{3} \mathrm{OH}$ ); IR $v_{\max }$ (film) 2929, 2857, 1717, 1642, 1456, 1192, $701 \mathrm{~cm}^{-1} ;{ }^{1} \mathrm{H}$ NMR (500 MHz, $d_{4}$-methanol) $\delta 7.17-7.28(\mathrm{~m}, 5 \mathrm{H}$, ArH), 5.48-5.58 (m, 2H, CH=CH), 4.68 (dd, J=9.1, 4.7 Hz, 1H, CHNH), 3.23 (dd, J = 14.0, 4.7 Hz, 1H, $\mathrm{CH}_{2} \mathrm{CHNH}$ ), 3.19 (d, J = 8.5 Hz, 1H, CH=CHCH), 2.99 (dd, J = 14.0, 9.1 Hz, $\left.1 \mathrm{H}, \mathrm{CH}_{2} \mathrm{CHNH}\right), 2.88\left(\mathrm{~d}, J=16.1 \mathrm{~Hz}, 1 \mathrm{H}, \mathrm{CH}_{2} \mathrm{CO}_{2} \mathrm{H}\right), 2.56\left(\mathrm{~d}, J=16.1 \mathrm{~Hz}, 1 \mathrm{H}, \mathrm{CH}_{2} \mathrm{CO}_{2} \mathrm{H}\right)$, 2.44 (td, $J=7.3,1.3 \mathrm{~Hz}, 4 \mathrm{H}, \mathrm{CH}_{2} \mathrm{C}(\mathrm{O}) \mathrm{CH}_{2}$ ), 1.97 (q, $J=6.4 \mathrm{~Hz}, 2 \mathrm{H}, \mathrm{CH}_{2} \mathrm{CH}=\mathrm{CH}$ ), 1.50$1.57\left(\mathrm{~m}, 4 \mathrm{H}, \mathrm{CH}_{2}\right), 1.27-1.34$ (brm, $\left.14 \mathrm{H}, \mathrm{CH}_{2}\right), 0.90$ (t, J=7.0 Hz, 3H, $\left.\mathrm{CH}_{3}\right)$ ppm; ${ }^{13} \mathrm{C}$ NMR (125 MHz, $d_{4}$-methanol) $\delta 214.5,175.7,174.3,173.8,173.5,138.2,137.5,130.4,129.4$, 127.9, 124.5, 77.9, 57.9, 54.8, 43.49, 43.48, 43.0, 38.3, 33.5, 32.9, 30.30, 30.27, 30.1, 29.90, 29.86, 24.9 24.8, 23.7, 14.4 ppm; HRMS (ESI) $\mathrm{m} / \mathrm{z}$ : Calcd for $\mathrm{C}_{31} \mathrm{H}_{46} \mathrm{NO} 9$ [M+H]+ 576.3173; Found 576.3171.

\section{Desmethylene analogue (17)}

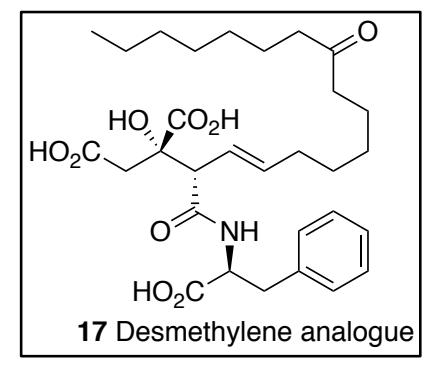

A solution of $\beta$-lactone 3 (50 $\mathrm{mg}, 0.153 \mathrm{mmol})$, alkene 4 (110 $\mathrm{mg}$, $0.461 \mathrm{mmol}$ ) and Grubbs-Hoveyda catalyst (12) (26 mg, 0.041 mmol) in $\mathrm{CH}_{2} \mathrm{Cl}_{2}(2 \mathrm{~mL})$ was heated under reflux for $3 \mathrm{~h}$ before being concentrated under reduced pressure. The crude material hexane) to afford cross metathesis product 13 (30 mg, contaminated with the alkene 4 homodimer and a similar byproduct). A solution of the $\beta$-lactone 13 mixture $(30 \mathrm{mg})$, Lphenylalanine-tert-butyl ester hydrochloride (15) (45 mg, $0.175 \mathrm{mmol}$ ) (15) and sodium-2ethylhexanoate (14) $(19 \mathrm{mg}, 0.114 \mathrm{mmol})$ in THF $(1.0 \mathrm{~mL})$ then under reflux for $19 \mathrm{~h}$. The solution was cooled to room temperature before ethyl acetate $(10 \mathrm{~mL})$ and water $(10 \mathrm{~mL})$ 
were added. The aqueous layer was further extracted with ethyl acetate $(3 \times 10 \mathrm{~mL})$, and the combined organic layers were washed with water $(10 \mathrm{~mL})$ and then brine $(10 \mathrm{~mL})$ before being concentrated under reduced pressure. The crude material was purified by flash chromatography (15\% ethyl acetate in hexane) and then HPLC (5 micron silica column $250 \times 10 \mathrm{~mm}, 20 \%$ ethyl acetate in hexane) to afford viridiofungin B tri-tert-butyl ester (16) contaminated with the byproduct $(56.2 \mathrm{mg}, 49 \%$ from 3 ). A portion of this (20 $\mathrm{mg}, 0.027 \mathrm{mmol})$ was dissolved in formic acid $(1.5 \mathrm{~mL})$ and the reaction mixture was stirred at room temperature for $1 \mathrm{~h} 15$ mins before the solvent was concentrated under reduced pressure. The crude material was purified by preparative reverse phase HPLC (LUNA $5 \mu$ C18 column 250×10 mm, 90\% methanol in $\mathrm{H}_{2} \mathrm{O}$ with $0.1 \%$ TFA (2 mL/min flow rate) to afford viridiofungin $\mathrm{B}(2)\left(R_{\mathrm{t}}=12.48 \mathrm{mins}, 6.1 \mathrm{mg}, 42 \%\right)$ and desmethylene analogue (17) ( $\left.R_{\mathrm{t}}=11.33 \mathrm{mins}, 6.4 \mathrm{mg}, 40 \%\right)$ as colorless amorphous solids. Data for 17: $[\alpha]_{D}^{24}=-14.9^{\circ}\left(c\right.$ 0.30, $\left.\mathrm{CH}_{3} \mathrm{OH}\right)$; IR $v_{\max }($ film) 2928, 2856, 2502, 1715, 1635, 1456, 1193, 973, 904, 798, 725, $701 \mathrm{~cm}^{-1}$; ${ }^{1} \mathrm{H}$ NMR (500 MHz, $d_{4}$-methanol) $\delta$ 7.18-7.29 (m, 5H, ArH), 5.48-5.59 (m, 2H, CH=CH), 4.68 (dd, J = 9.0, 4.8 Hz, 1H, CHNH), 3.23 (dd, J=14.0, 4.6 $\mathrm{Hz}, 1 \mathrm{H}, \mathrm{CH}_{2} \mathrm{CHNH}$ ), 3.19 (d, J = $\left.8.4 \mathrm{~Hz}, 1 \mathrm{H}, \mathrm{CH}=\mathrm{CHCH}\right), 2.99$ (dd, $J=14.0,9.1 \mathrm{~Hz}, 1 \mathrm{H}$, $\mathrm{CH}_{2} \mathrm{CHNH}$ ), $2.88\left(\mathrm{~d}, J=16.2 \mathrm{~Hz}, 1 \mathrm{H}, \mathrm{CH}_{2} \mathrm{CO}_{2} \mathrm{H}\right), 2.56\left(\mathrm{~d}, J=16.2 \mathrm{~Hz}, 1 \mathrm{H}, \mathrm{CH}_{2} \mathrm{CO}_{2} \mathrm{H}\right)$, $2.44\left(\mathrm{t}, J=7.3 \mathrm{~Hz}, 4 \mathrm{H}, \mathrm{CH}_{2} \mathrm{C}(\mathrm{O}) \mathrm{CH}_{2}\right), 1.96-2.00\left(\mathrm{~m}, 2 \mathrm{H}, \mathrm{CH}_{2} \mathrm{CH}=\mathrm{CH}\right), 1.49-1.57(\mathrm{~m}, 4 \mathrm{H}$, $\mathrm{CH}_{2}$ ), 1.25-1.347(br m, 12H, $\left.\mathrm{CH}_{2}\right), 0.90$ (t, J = 6.9 Hz, 3H, $\left.\mathrm{CH}_{3}\right) \mathrm{ppm} ;{ }^{33} \mathrm{C}$ NMR (125 MHz, $d_{4}$-methanol) $\delta 214.4,175.7,174.3,173.8,173.5,138.2,137.4,130.4,129.4,127.9,124.6$, 77.9, 57.9, 54.8, 43.5, 43.4, 43.0, 38.2, 33.3, 32.9, 30.30, 30.27, 29.8, 29.6, 24.9 24.7, 23.7, 14.4 ppm; HRMS (ESI) $\mathrm{m} / \mathrm{z}$ : Calcd for $\mathrm{C}_{30} \mathrm{H}_{44} \mathrm{NO}_{9}[\mathrm{M}+\mathrm{H}]^{+}$562.3016; Found 562.3016 .

\section{Viridiofungin A tri-t-butyl ester 20}

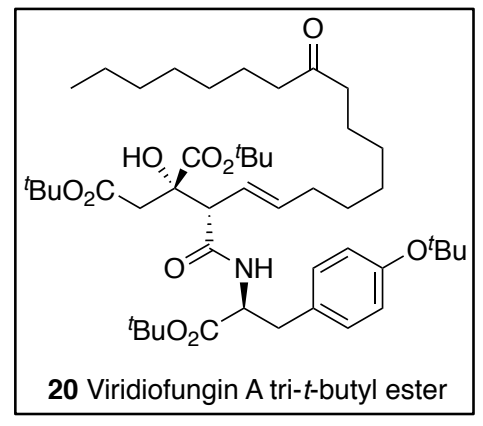

A solution of $\beta$-lactone 3 (100 $\mathrm{mg}, 0.307 \mathrm{mmol})$, alkene 4 (110 $\mathrm{mg}, 0.460 \mathrm{mmol}$ ) and Grubbs-Hoveyda catalyst (12) (38 mg, $0.061 \mathrm{mmol})$ in $\mathrm{CH}_{2} \mathrm{Cl}_{2}(4 \mathrm{~mL})$ was heated under reflux for $2 \mathrm{~h}$ before being concentrated under reduced pressure. The crude material was quickly purified by flash chromatography (5-10\% EtOAc in hexane) to afford cross metathesis product 13 (107.8 $\mathrm{mg}$, contaminated with alkene dimer). A solution of the $\beta$-lactone 13 mixture (45 mg), Ltyrosine(tert-butyl)-tert-butyl ester hydrochloride (19) $(66 \mathrm{mg}, 0.20 \mathrm{mmol}$ ) and sodium-2- 
ethylhexanoate (14) (22 mg, $0.11 \mathrm{mmol})$ in THF (1.25 mL) was heated under reflux for 24 h. The solution was cooled to room temperature before ethyl acetate $(15 \mathrm{~mL})$ and water $(15 \mathrm{~mL})$ were added. The aqueous layer was extracted into ethyl acetate $(3 \times 15 \mathrm{~mL})$, and the combined organic layers were washed with water $(15 \mathrm{~mL})$ and then brine $(15 \mathrm{~mL})$ before being concentrated. The crude material was purified by flash chromatography (10$15 \%$ ethyl acetate in hexane) to afford viridiofungin A tri-t-butyl ester (20) (48 mg, 46\% from 3) as a colorless oil. $[\alpha]_{D}^{22}=-10.7^{\circ}\left(c 0.80, \mathrm{CH}_{2} \mathrm{Cl}_{2}\right)$; IR $v_{\max }$ (film) 3381, 2977, 2931, 2857, 1733, 1675, 1507, 1457, 1393, 1367, 1237, 1156, 972, 900, 847, $754 \mathrm{~cm}^{-1}$; ${ }^{1} \mathrm{H}$ NMR $\left(500 \mathrm{MHz}, \mathrm{CDCl}_{3}\right) \delta 7.10(\mathrm{~d}, J=8.3 \mathrm{~Hz}, 2 \mathrm{H}, \mathrm{ArH}), 7.04(\mathrm{~d}, J=8.3 \mathrm{~Hz}, 1 \mathrm{H}, \mathrm{NH}), 6.88(\mathrm{~d}, J$ $=8.3 \mathrm{~Hz}, 2 \mathrm{H}, \mathrm{ArH}), 5.63(\mathrm{dt}, J=14.8,7.1 \mathrm{~Hz}, 1 \mathrm{H}, \mathrm{CH}=\mathrm{CHCHC}(\mathrm{O})), 5.46$ (dd, J = 15.1, 9.4 $\mathrm{Hz}, 1 \mathrm{H}, \mathrm{CH}=\mathrm{CHCHC}(\mathrm{O})), 4.68(\mathrm{q}, J=7.2 \mathrm{~Hz}, 1 \mathrm{H}, \mathrm{CHNH}), 4.22(\mathrm{~s}, 1 \mathrm{H}, \mathrm{OH}), 3.09(\mathrm{~d}, J=$ $9.4 \mathrm{~Hz}, 1 \mathrm{H}, \mathrm{CH}=\mathrm{CHCHC}(\mathrm{O})$ ), $3.04\left(\mathrm{dd}, J=6.0,2.9 \mathrm{~Hz}, 2 \mathrm{H}, \mathrm{CH}_{2} \mathrm{CHNH}\right), 2.75$ (d, J = 16.5 $\left.\mathrm{Hz}, 1 \mathrm{H}, \mathrm{CH}_{2} \mathrm{CO}_{2}{ }^{\mathrm{t}} \mathrm{Bu}\right), 2.61\left(\mathrm{~d}, J=16.6 \mathrm{~Hz}, 1 \mathrm{H}, \mathrm{CH}_{2} \mathrm{CO}_{2}{ }^{\mathrm{t}} \mathrm{Bu}\right), 2.36(\mathrm{td}, J=7.3,4.3 \mathrm{~Hz}, 4 \mathrm{H}$, $\left.\mathrm{CH}_{2} \mathrm{C}(\mathrm{O}) \mathrm{CH}_{2}\right), 1.94-1.98\left(\mathrm{~m}, 2 \mathrm{H}, \mathrm{CH}_{2} \mathrm{CH}=\mathrm{CH}\right), 1.53\left(\mathrm{dq}, J=14.9,7.6 \mathrm{~Hz}, 4 \mathrm{H}, \mathrm{CH}_{2}\right), 1.43$ and 1.44 (2x s overlapping, $\left.18 \mathrm{H},{ }^{t} \mathrm{Bu}\right), 1.36\left(\mathrm{~s}, 9 \mathrm{H},{ }^{t} \mathrm{Bu}\right), 1.31\left(\mathrm{~s}, 9 \mathrm{H},{ }^{t} \mathrm{Bu}\right), 1.26(\mathrm{br} \mathrm{m}$, $\left.14 \mathrm{H}, \mathrm{CH}_{2}\right), 0.87\left(\mathrm{t}, J=6.8 \mathrm{~Hz}, 3 \mathrm{H}, \mathrm{CH}_{3}\right) \mathrm{ppm} ;{ }^{13} \mathrm{C} \mathrm{NMR}\left(125.5 \mathrm{MHz}, \mathrm{CDCl}_{3}\right) \delta 211.8$, $172.1,170.5,170.2,169.5,154.3,137.0,131.5,130.2,124.2,122.7,83.2,82.1,81.6$, 78.6, 75.7, 58.4, 54.0, 43.0, 42.9, 42.6, 37.8, 32.7, 31.8, 29.4, 29.22, 29.16, 29.0, 28.2, 28.10, 28.07, 24.0, 23.9, 22.8, 14.2 ppm; HRMS (ESI) $\mathrm{m} / \mathrm{z}$ : Calcd for $\mathrm{C}_{47} \mathrm{H}_{78} \mathrm{NO}_{10}[\mathrm{M}+\mathrm{H}]^{+}$ 816.5626; Found 816.5671.

\section{Viridiofungin A (1)}

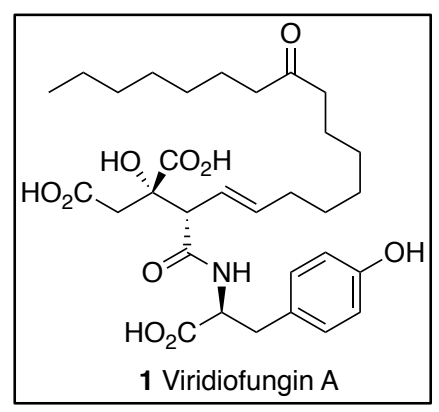

Viridiofungin A tri-t-butyl ester (20) $(15 \mathrm{mg}, 0.018 \mathrm{mmol}$ ) was dissolved in formic acid $(1 \mathrm{~mL})$. The reaction mixture was stirred at room temperature for $16.5 \mathrm{~h}$ before being concentrated under reduced pressure to afford pure viridiofungin $A$ (1) as an amorphous solid (10.5 mg, 96\%). $[\alpha]_{D}^{22}=-24.7^{\circ}\left(c 0.45, \mathrm{CH}_{3} \mathrm{OH}\right)$. Lit. $^{1}[\alpha]_{D}^{25}=-18.2^{\circ}\left(c\right.$ 2.37, $\left.\mathrm{CH}_{3} \mathrm{OH}\right)$; IR $\mathrm{V}_{\max }$ (film) 3735, 3309, 2926, 2856, 1718, 1647, 1517, 1371, 1225, 974, $841 \mathrm{~cm}^{-1}$; ${ }^{1} \mathrm{H}$ NMR $\left(500 \mathrm{MHz}, d_{4-}\right.$ methanol) $\delta 7.03(\mathrm{~d}, J=8.4 \mathrm{~Hz}, 2 \mathrm{H}, \mathrm{ArH}), 6.68(\mathrm{~d}, J=8.4 \mathrm{~Hz}, 2 \mathrm{H}, \mathrm{ArH}), 5.49-5.58(\mathrm{~m}, 2 \mathrm{H}$, $\mathrm{CH}=\mathrm{CH}), 4.61(\mathrm{dd}, J=8.6,4.7 \mathrm{~Hz}, 1 \mathrm{H}, \mathrm{CHNH}), 3.22(\mathrm{~d}, J=8.2 \mathrm{~Hz}, 1 \mathrm{H}, \mathrm{CH}=\mathrm{CHCH}), 3.11$ (dd, $J=14.1,4.6 \mathrm{~Hz}, 1 \mathrm{H}, \mathrm{CH}_{2} \mathrm{CHNH}$ ), $2.91\left(\mathrm{~d}, J=15.4 \mathrm{~Hz}, 1 \mathrm{H}, \mathrm{CH}_{2} \mathrm{CO}_{2}{ }^{\mathrm{B} u}\right.$ ), 2.90 (dd, $J=$ 13.7, 9.1 Hz, $\mathrm{CH}_{2} \mathrm{CHNH}$ ), $2.63\left(\mathrm{~d}, J=16.1 \mathrm{~Hz}, 1 \mathrm{H}, \mathrm{CH}_{2} \mathrm{CO}_{2} \mathrm{Bu}^{\mathrm{B}}\right), 2.44$ (td, $J=7.3,1.8 \mathrm{~Hz}$, 
$\left.4 \mathrm{H}, \mathrm{CH}_{2} \mathrm{C}(\mathrm{O}) \mathrm{CH}_{2}\right), 1.97-1.99\left(\mathrm{~m}, 2 \mathrm{H}, \mathrm{CH}_{2} \mathrm{CH}=\mathrm{CH}\right), 1.52-1.55\left(\mathrm{~m}, 4 \mathrm{H}, \mathrm{CH}_{2}\right), 1.27-1.34$ (brm, $14 \mathrm{H}, \mathrm{CH}_{2}$ ), 0.90 (t, $\left.J=6.9 \mathrm{~Hz}, 3 \mathrm{H}, \mathrm{CH}_{3}\right) \mathrm{ppm} ;{ }^{13} \mathrm{C} \mathrm{NMR}\left(125 \mathrm{MHz}, d_{4}\right.$-methanol) $\delta$ 214.6, 175.7, 174.5, 173.9, 173.6, 157.4, 137.6, 131.4, 128.7, 124.5, 116.2, 77.9, 57.7, 55.1, 43.5, 43.1, 37.5, 33.5, 32.9, 30.29, 30.27, 30.1, 29.9, 29.8, 24.9, 24.8, 23.7, 14.4 ppm; HRMS (ESI) m/z: Calcd for $\mathrm{C}_{31} \mathrm{H}_{46} \mathrm{NO}_{10}[\mathrm{M}+\mathrm{H}]^{+} 592.3122$; Found 592.3120 . 


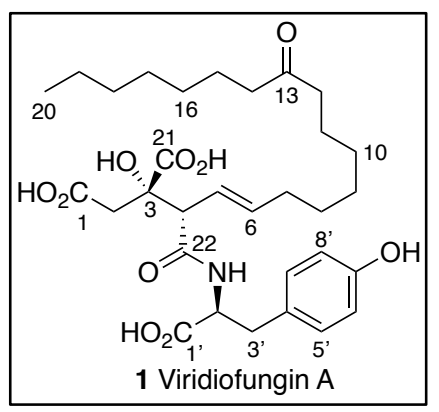

NMR data for natural and synthetic viridiofungin $A$ (1)

\begin{tabular}{|c|c|c|c|c|c|}
\hline Proton & $\begin{array}{l}\text { Natural }{ }^{3} 1 \\
\delta(\mathrm{m}, J \mathrm{~Hz})\end{array}$ & $\begin{array}{l}\text { Synthetic } 1 \\
\delta(\mathrm{m}, J \mathrm{~Hz})\end{array}$ & Carbon & $\begin{array}{c}\text { Natural }^{3} 1 \\
\delta \\
\end{array}$ & $\begin{array}{c}\text { Synthetic } 1 \\
\delta\end{array}$ \\
\hline $2 a$ & $2.62(\mathrm{~d}, 16.3)$ & $2.63(\mathrm{~d}, 16.1)$ & 1 & 173.6 & 173.6 \\
\hline $2 b$ & $2.91(\mathrm{~d}, 16.3)$ & $2.91(\mathrm{~d}, 16.1)$ & 2 & 43.1 & 43.1 \\
\hline 4 & $3.22(d, 8.5)$ & $3.22(d, 8.2)$ & 3 & 77.9 & 77.9 \\
\hline $5 / 6$ & $5.53(\mathrm{~m})$ & $5.53(\mathrm{~m})$ & 4 & 57.8 & 57.7 \\
\hline 7 & $1.98(\mathrm{~m})$ & 1.98 (m) & 5 & 137.6 & 137.6 \\
\hline $\begin{array}{l}8-10 \\
16-19\end{array}$ & 1.29 (brm) & 1.29 (brm) & 6 & 124.5 & 124.5 \\
\hline $11 / 15$ & $1.53(\mathrm{~m})$ & $1.53(\mathrm{~m})$ & 7 & 33.5 & 33.5 \\
\hline $12 / 14$ & $2.44(t, 7.2)$ & $\begin{array}{c}2.44(\mathrm{td}, 7.3 \\
1.8)\end{array}$ & $\begin{array}{l}8-10 \\
16-18\end{array}$ & $\begin{array}{c}29.8,29.9, \\
30.1,30.26 \\
30.29,32.9\end{array}$ & $\begin{array}{l}29.8,29.9, \\
30.1,30.27 \\
30.29,32.9\end{array}$ \\
\hline 20 & $0.90(t, 7.0)$ & $0.90(t, 6.9)$ & 11 & 24.8 & 24.8 \\
\hline $2^{3}$ & $\begin{array}{c}4.61 \text { (dd, 5.0, } \\
8.7)\end{array}$ & $\begin{array}{c}4.61(\mathrm{dd}, 4.7, \\
8.6)\end{array}$ & $12 / 14$ & 43.5 & 43.5 \\
\hline $3 a^{\prime}$ & $\begin{array}{c}2.89(\mathrm{dd}, 8.7 \\
14.3)\end{array}$ & $\begin{array}{c}2.90(\mathrm{dd}, 9.1 \\
13.7)\end{array}$ & 13 & 214.6 & 214.6 \\
\hline $3 b^{\prime}$ & $\begin{array}{c}3.11(\mathrm{dd}, 5.3 \\
14.3)\end{array}$ & $\begin{array}{c}3.11(d d, 4.6, \\
14.1)\end{array}$ & 15 & 24.9 & 24.9 \\
\hline $5^{\prime} / 9^{\prime}$ & $7.03(\mathrm{~d}, 8.8)$ & $7.03(d, 8.4)$ & 19 & 23.7 & 23.7 \\
\hline \multirow[t]{10}{*}{$6^{\prime} / 8^{\prime}$} & $6.68(\mathrm{~d}, 8.8)$ & $6.68(\mathrm{~d}, 8.4)$ & 20 & 14.4 & 14.4 \\
\hline & & & 21 & 175.8 & 175.7 \\
\hline & & & 22 & 173.8 & 173.9 \\
\hline & & & $1^{\prime}$ & 174.5 & 174.5 \\
\hline & & & $2^{\prime}$ & 55.1 & 55.1 \\
\hline & & & $3^{\prime}$ & 37.5 & 37.5 \\
\hline & & & $4^{\prime}$ & 128.7 & 128.7 \\
\hline & & & $5^{\prime} / 9^{\prime}$ & 131.4 & 131.4 \\
\hline & & & 6'/8' & 116.2 & 116.2 \\
\hline & & & $7^{\prime}$ & 157.4 & 157.4 \\
\hline
\end{tabular}

(3) Harris, G. H.; Turner Jones, E. T.; Meinz, M. S.; Nallin-Omstead, M.; Helms, G. L.; Bills, G. F.; Zink, D.; Wilson, K. E. Tetrahedron Lett. 1993, 34, 5235-5238. 


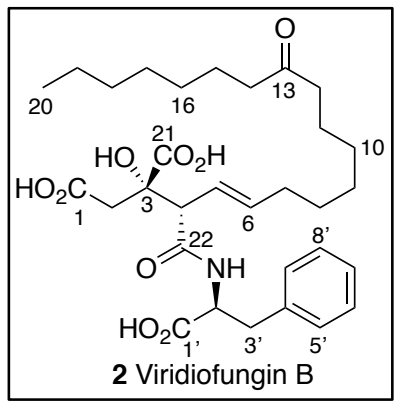

NMR data for natural and synthetic viridiofungin B (2)

\begin{tabular}{|c|c|c|c|c|c|}
\hline \multicolumn{3}{|c|}{${ }^{1} \mathrm{H}$ NMR spectra $d_{4}$-methanol } & \multirow[b]{2}{*}{ Carbon } & \multirow[b]{2}{*}{$\begin{array}{c}\text { Natural }^{3} 2 \\
\delta\end{array}$} & \multirow[b]{2}{*}{$\begin{array}{c}\text { Synthetic } 2 \\
\delta\end{array}$} \\
\hline Proton & $\begin{array}{l}\text { Natural }^{3} 2 \\
\delta(\mathrm{m}, J \mathrm{~Hz})\end{array}$ & $\begin{array}{l}\text { Synthetic } 2 \\
\delta \text { (m, J Hz) }\end{array}$ & & & \\
\hline $2 \mathrm{a}$ & $2.56(d, 16.0)$ & $2.56(\mathrm{~d}, 16.1)$ & 1 & 173.5 & 173.5 \\
\hline $2 b$ & $2.88(\mathrm{~d}, 16.0)$ & $2.88(d, 16.1)$ & 2 & 43.0 & 43.0 \\
\hline 4 & $3.19(\mathrm{~d}, 8.4)$ & $3.19(\mathrm{~d}, 8.5)$ & 3 & 77.9 & 77.9 \\
\hline $5 / 6$ & $5.54(\mathrm{~m})$ & $5.53(\mathrm{~m})$ & 4 & 57.9 & 57.9 \\
\hline 7 & $1.97(\mathrm{~m})$ & $1.97(q, 6.4)$ & 5 & 137.5 & 137.5 \\
\hline $\begin{array}{l}8-10 \\
16-19\end{array}$ & 1.29 (brm) & 1.30 (brm) & 6 & 124.5 & 124.5 \\
\hline $11 / 15$ & $1.53(\mathrm{~m})$ & $1.54(\mathrm{~m})$ & 7 & 33.5 & 33.5 \\
\hline $12 / 14$ & $2.44(\mathrm{t}, 6.6)$ & $\begin{array}{c}2.44(\mathrm{td}, 1.3 \\
7.3)\end{array}$ & $\begin{array}{l}8-10 \\
16-18\end{array}$ & $\begin{array}{l}29.8,29.9, \\
30.1,30.2, \\
30.3,32.9\end{array}$ & $\begin{array}{c}29.86,29.90 \\
30.1,30.27 \\
30.30,32.9\end{array}$ \\
\hline 20 & $0.90(\mathrm{t}, 7.0)$ & $0.90(\mathrm{t}, 7.0)$ & 11 & 24.8 & 24.8 \\
\hline $2^{\prime}$ & $\begin{array}{c}4.69(\mathrm{dd}, 4.9 \\
9.4)\end{array}$ & $\begin{array}{c}4.68(d d, 4.7 \\
9.1)\end{array}$ & $12 / 14$ & $43.5(2)$ & $43.48,43.49$ \\
\hline $3 a^{\prime}$ & $\begin{array}{c}2.98(\mathrm{dd}, 9.4 \\
14.3)\end{array}$ & $\begin{array}{c}2.99(\mathrm{dd}, 9.1 \\
14.0)\end{array}$ & 13 & 214.5 & 214.5 \\
\hline $3 b^{3}$ & $\begin{array}{c}3.24(d d, 5.0 \\
14.3)\end{array}$ & $\begin{array}{c}3.23(\mathrm{dd}, 4.7, \\
14.0)\end{array}$ & 15 & 24.9 & 24.9 \\
\hline \multirow[t]{11}{*}{$5^{\prime}-9^{\prime}$} & $7.24(\mathrm{~m})$ & $7.23(\mathrm{~m})$ & 19 & 23.7 & 23.7 \\
\hline & & & 20 & 14.4 & 14.4 \\
\hline & & & 21 & 175.6 & 175.7 \\
\hline & & & 22 & 173.8 & 173.8 \\
\hline & & & $1^{\prime}$ & 174.3 & 174.3 \\
\hline & & & $2^{\prime}$ & 54.8 & 54.8 \\
\hline & & & $3^{\prime}$ & 38.2 & 38.3 \\
\hline & & & 4' & 127.9 & 127.9 \\
\hline & & & $5^{\prime} / 9^{\prime}$ & $130.4(2)$ & 130.4 \\
\hline & & & $6^{\prime} / 8^{\prime}$ & $129.4(2)$ & 129.4 \\
\hline & & & 7 & 138.2 & 138.2 \\
\hline
\end{tabular}




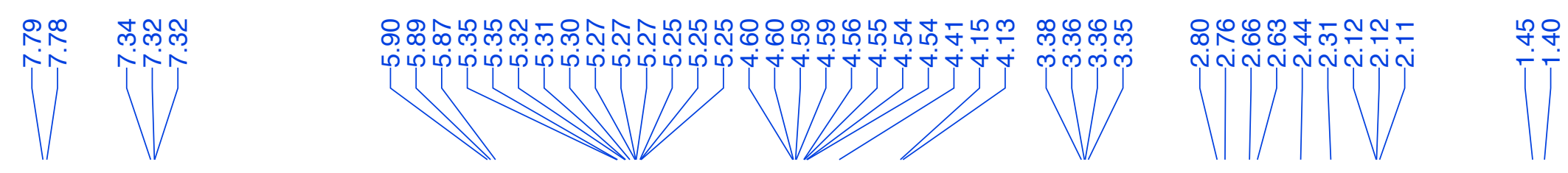

${ }^{1} \mathrm{H}$ NMR spectrum $\left(600 \mathrm{MHz}, \mathrm{CDCl}_{3}\right.$ )
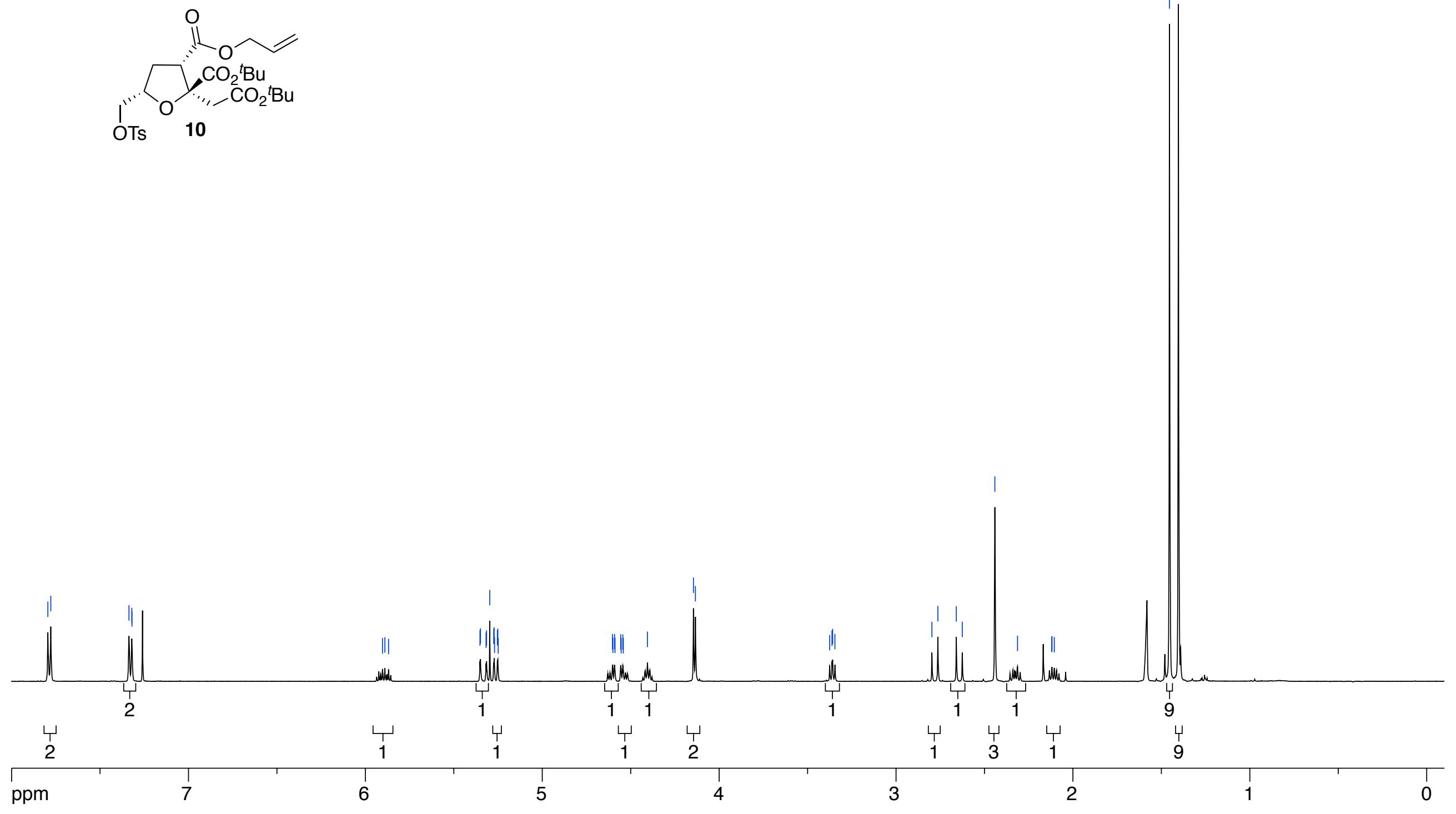


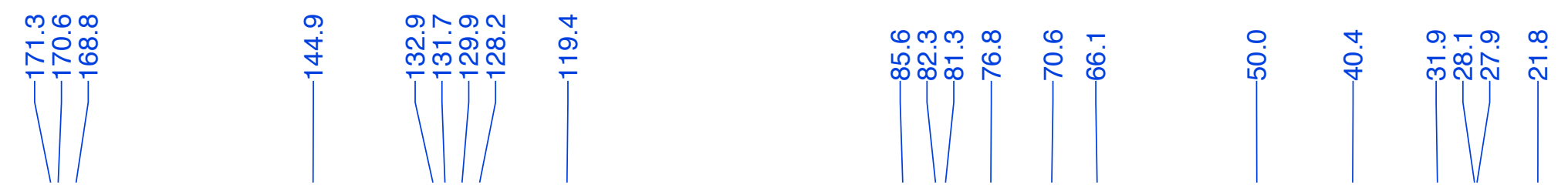

${ }^{13} \mathrm{C}$ NMR spectrum (100 MHz, $\mathrm{CDCl}_{3}$ )
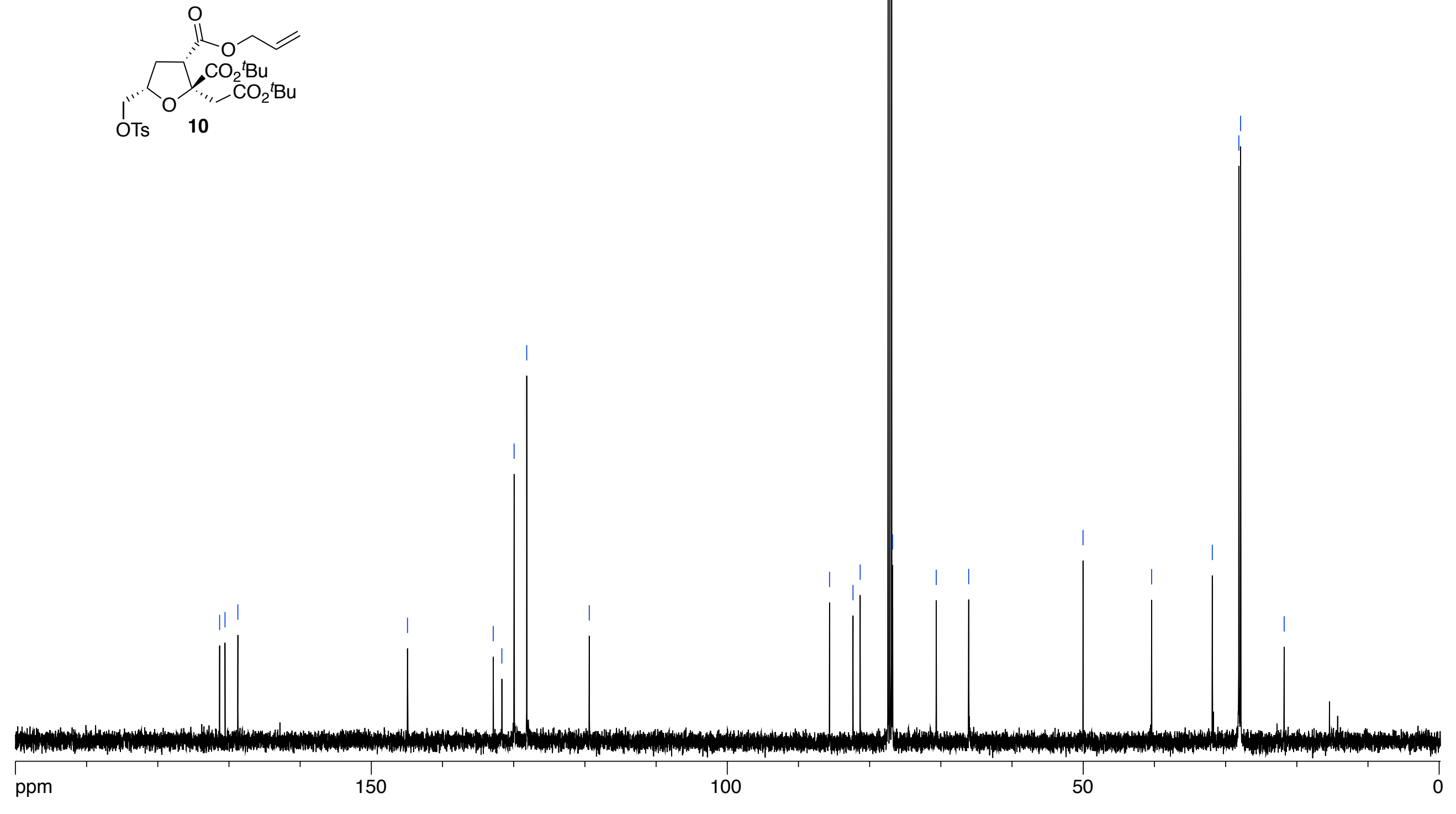


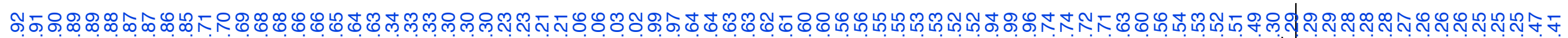

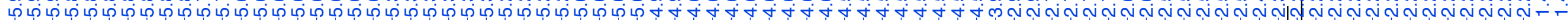

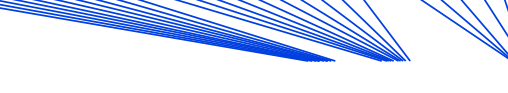

${ }^{1} \mathrm{H}$ NMR spectrum $\left(600 \mathrm{MHz}, \mathrm{CDCl}_{3}\right)$
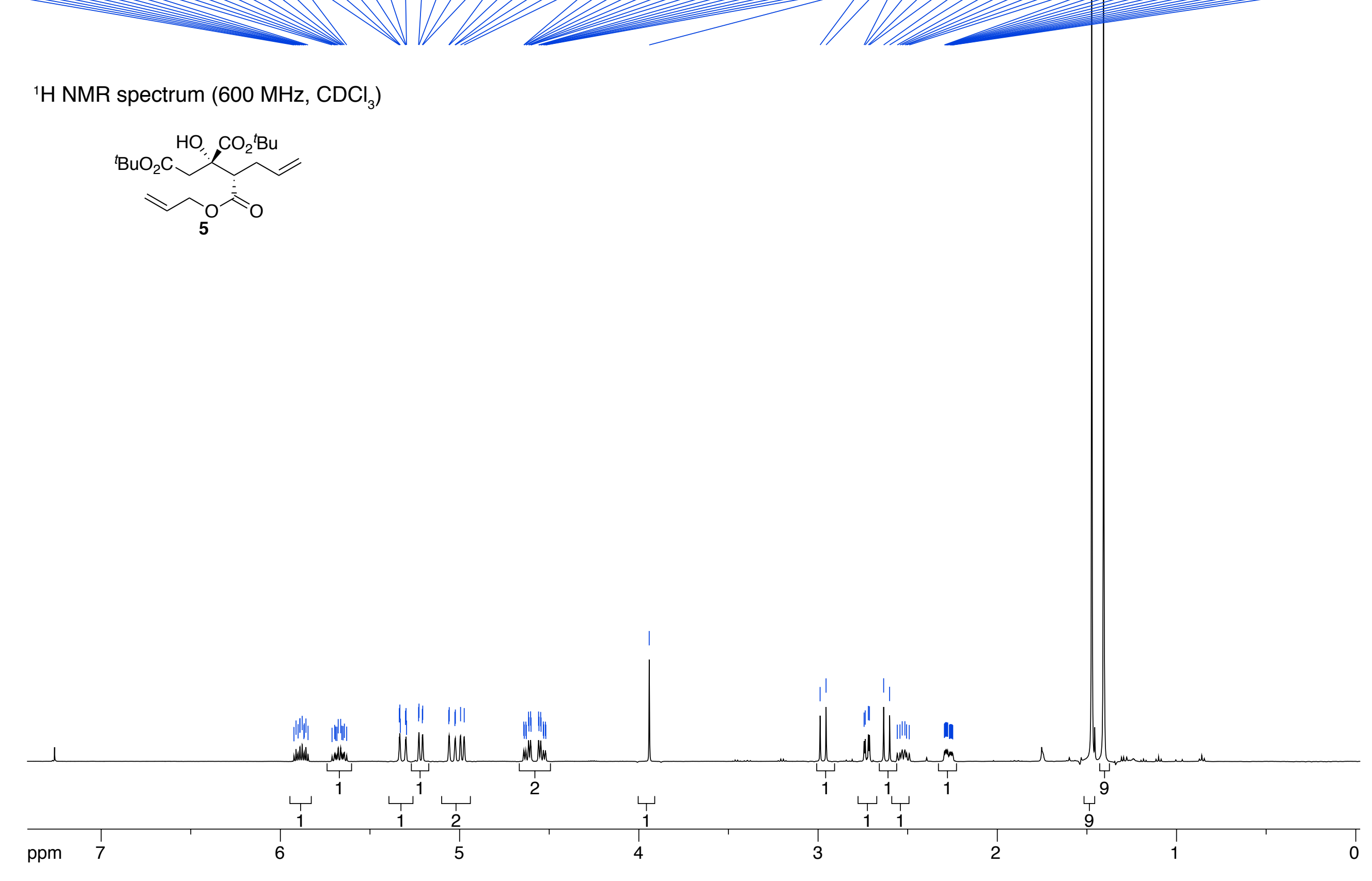

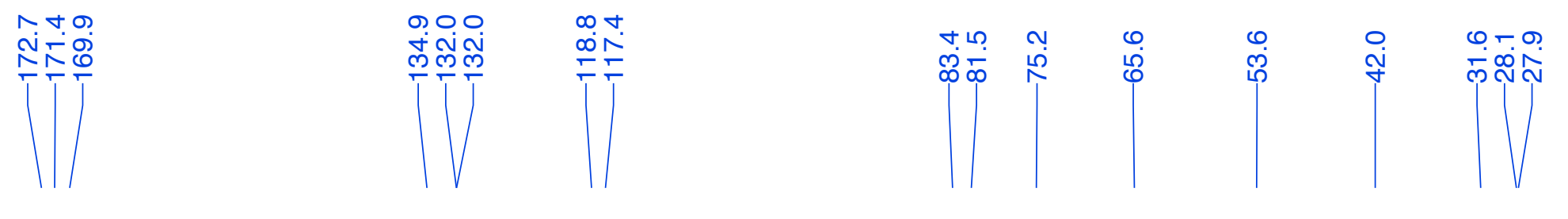

${ }^{13} \mathrm{C}$ NMR spectrum (125.5 MHz, $\mathrm{CDCl}_{3}$ )
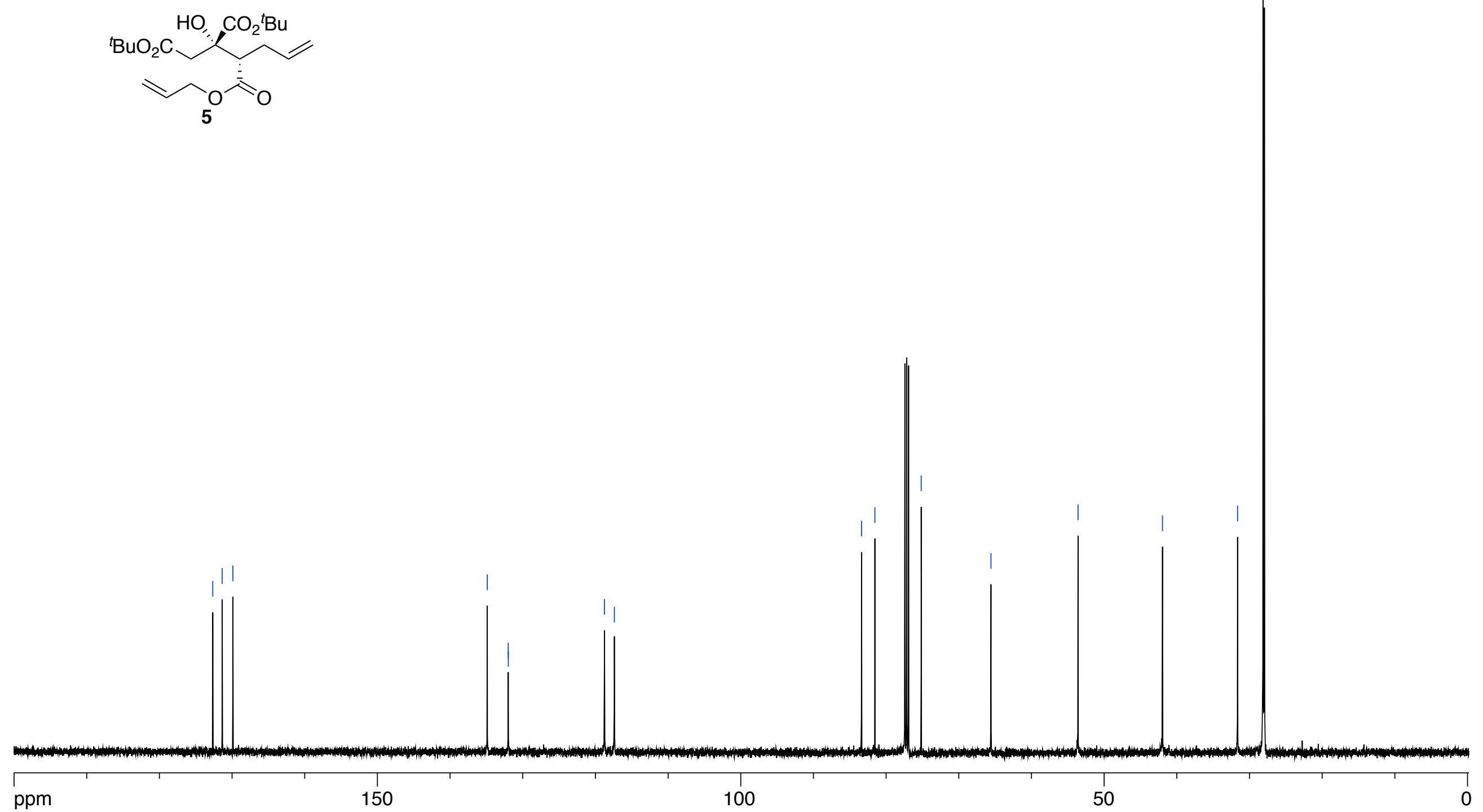


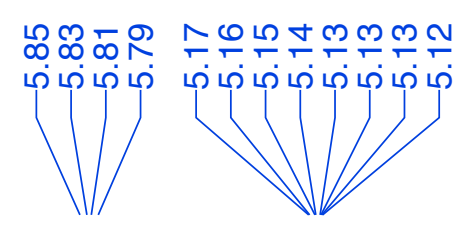

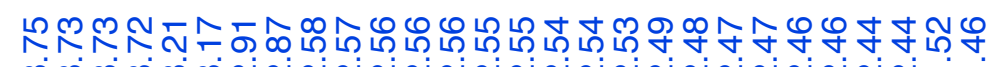
एறp

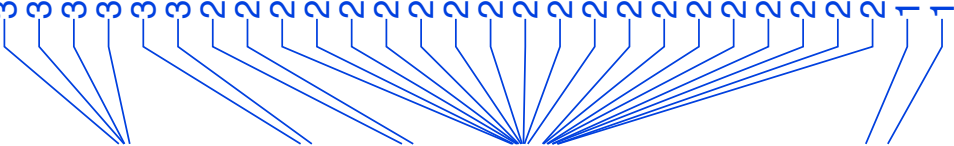

${ }^{1} \mathrm{H}$ NMR spectrum $\left(500 \mathrm{MHz}, \mathrm{CDCl}_{3}\right)$<smiles>C=CC[C@H]1C(=O)OC1(C(=O)OC(C)(C)C)C(=O)OC(C)(C)C</smiles>

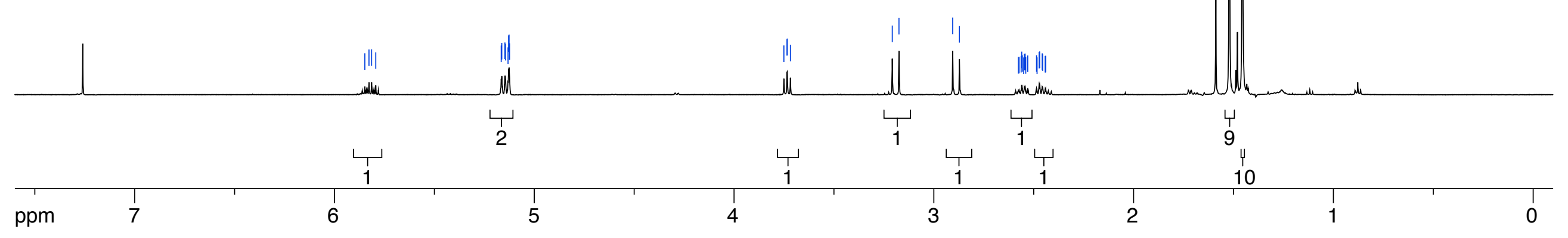




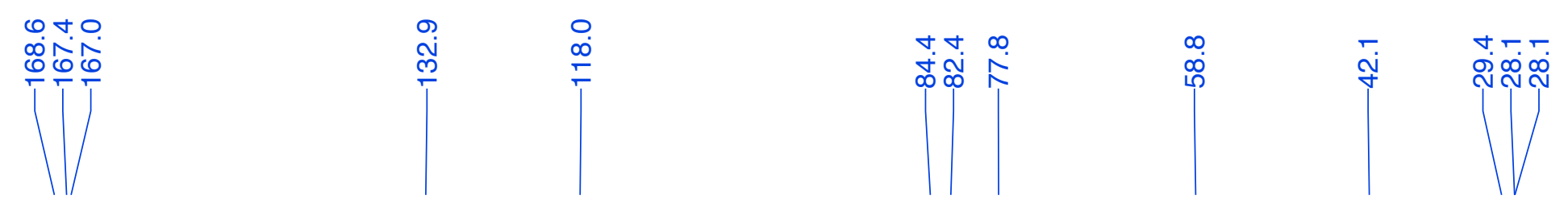

${ }^{13} \mathrm{C}$ NMR spectrum (125.5 MHz, $\mathrm{CDCl}_{3}$ )
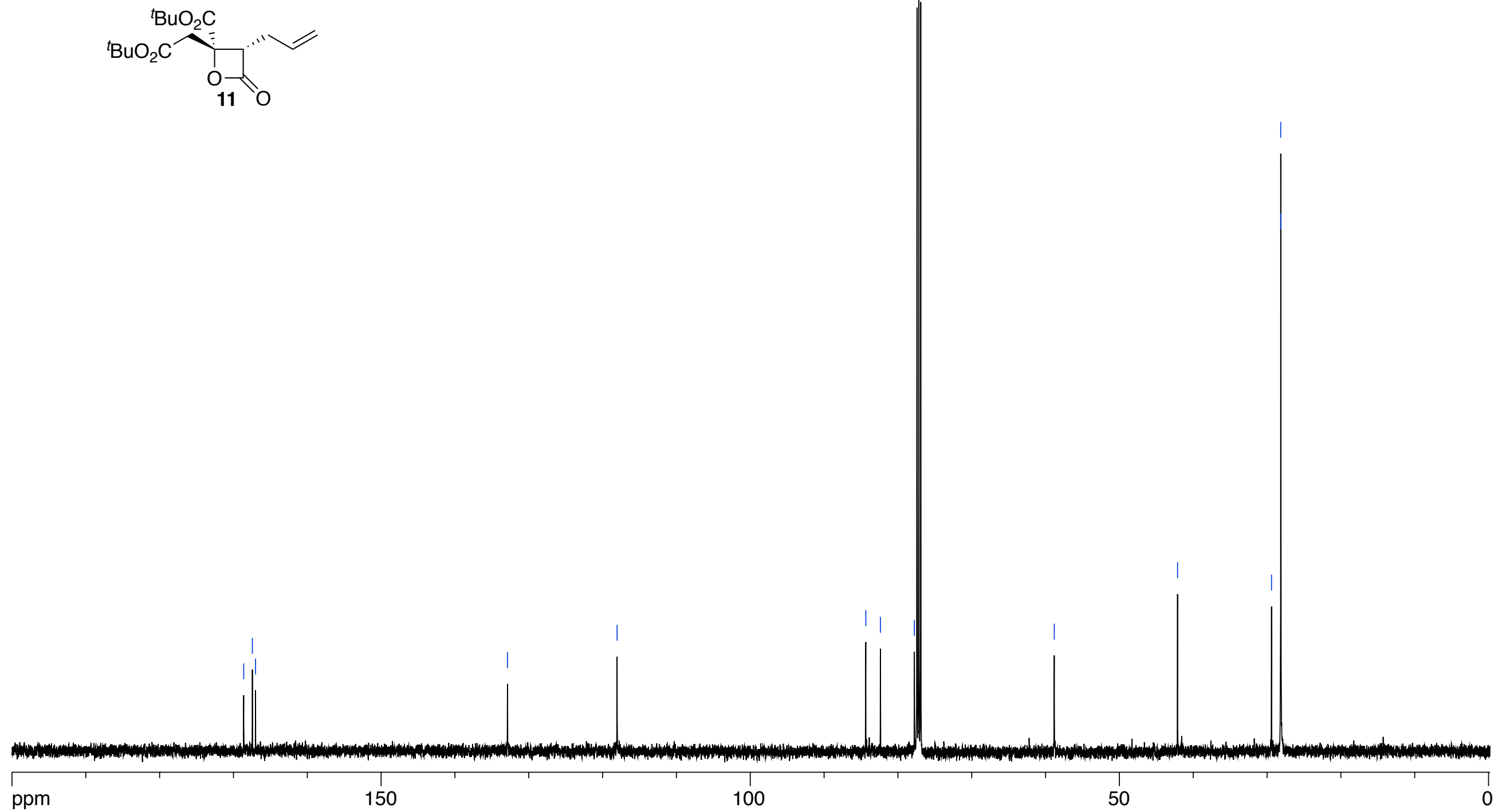


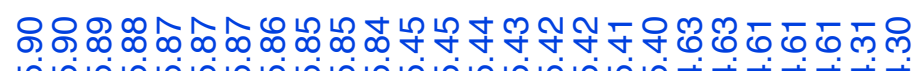

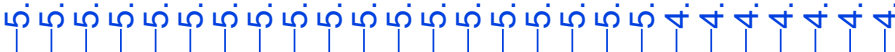

S20

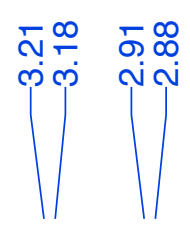

${ }^{1} \mathrm{H}$ NMR spectrum $\left(500 \mathrm{MHz}, \mathrm{CDCl}_{3}\right)$

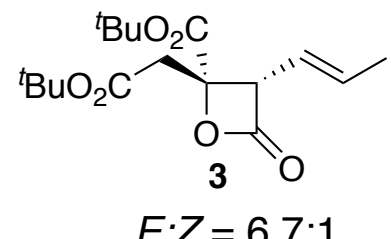

$E: Z=6.7: 1$

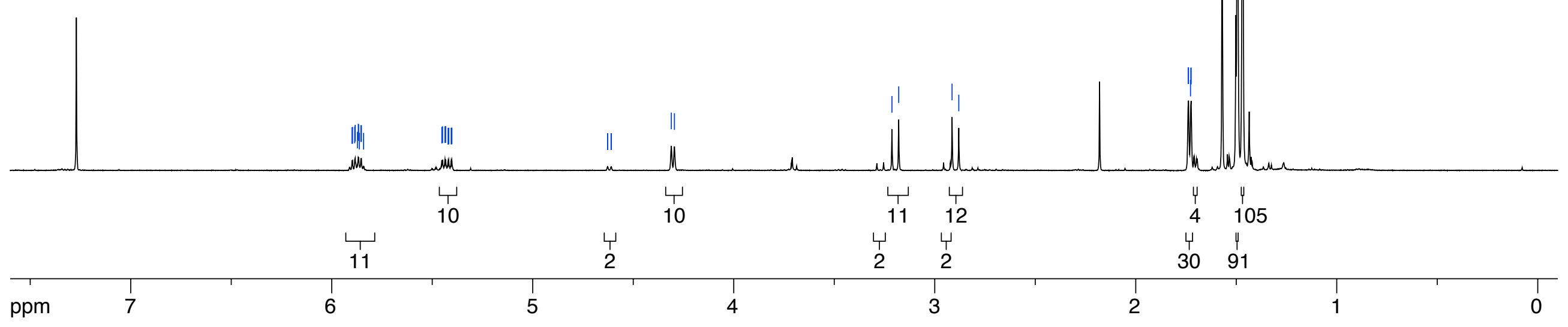




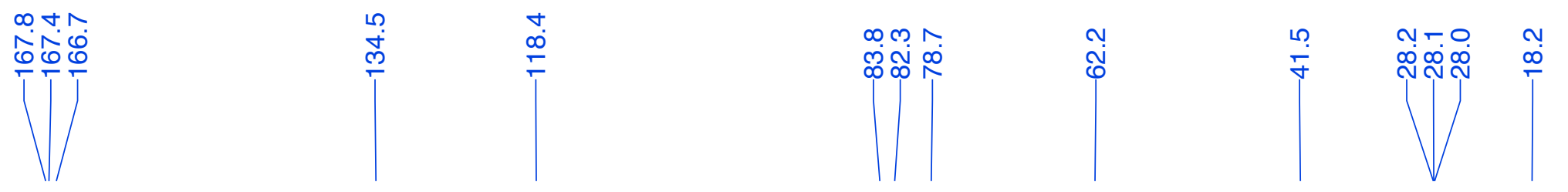

${ }^{13} \mathrm{C}$ NMR spectrum (125.5 MHz, $\mathrm{CDCl}_{3}$ )
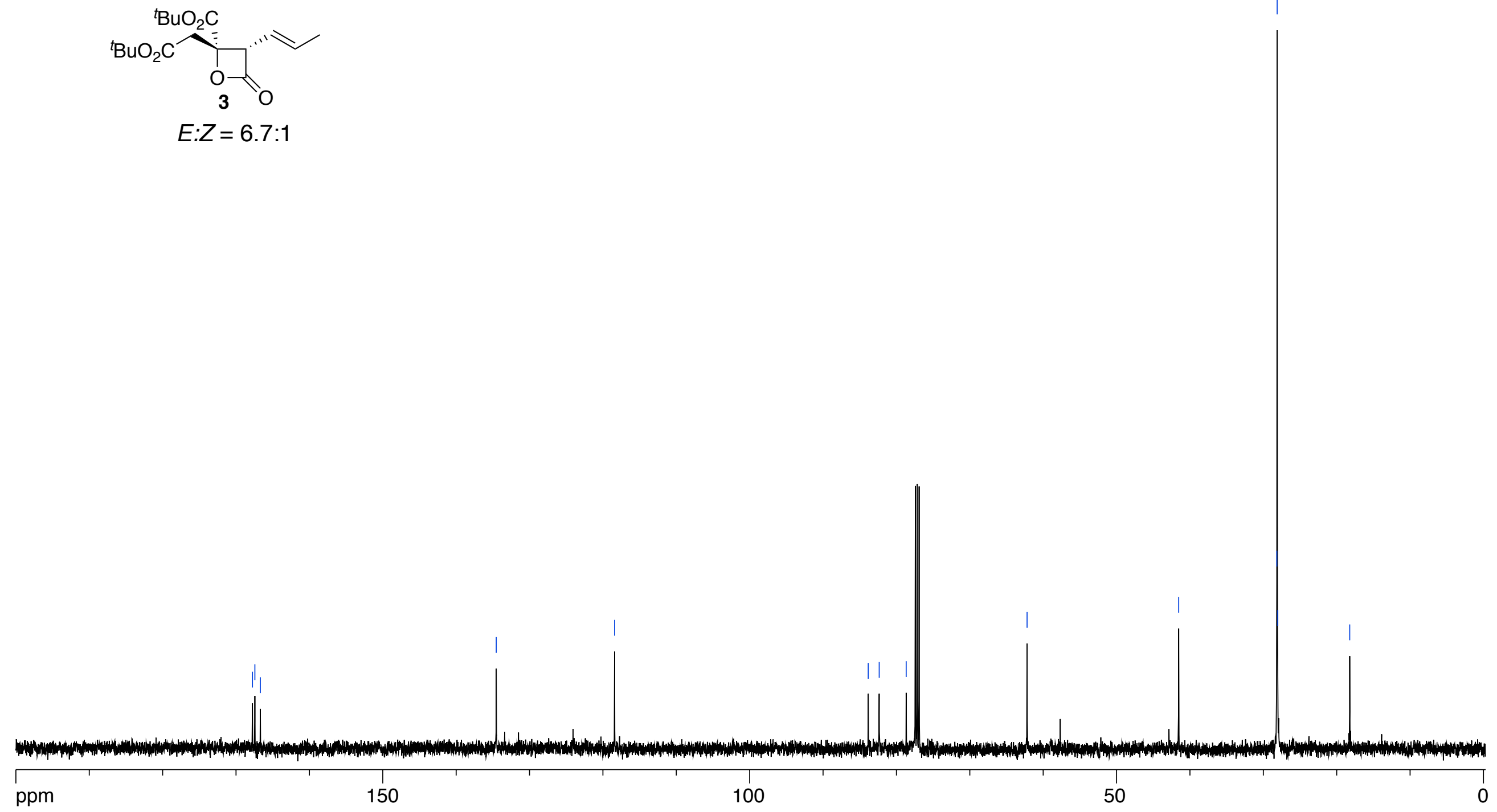


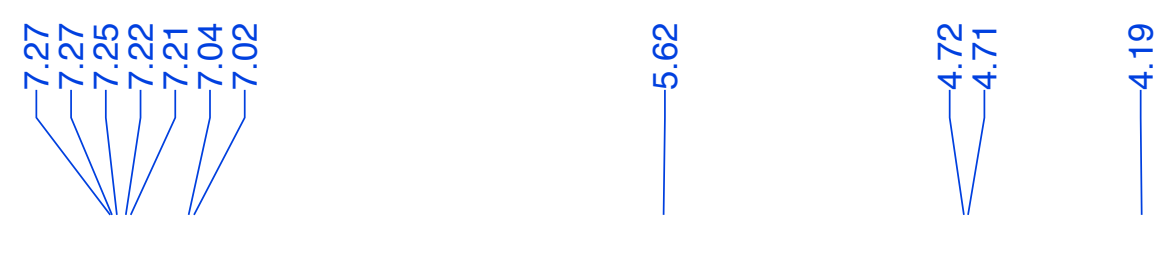

${ }^{1} \mathrm{H}$ NMR spectrum $\left(500 \mathrm{MHz}, \mathrm{CDCl}_{3}\right.$ )

16 Viridiofungin $B$ tri-t-butyl ester

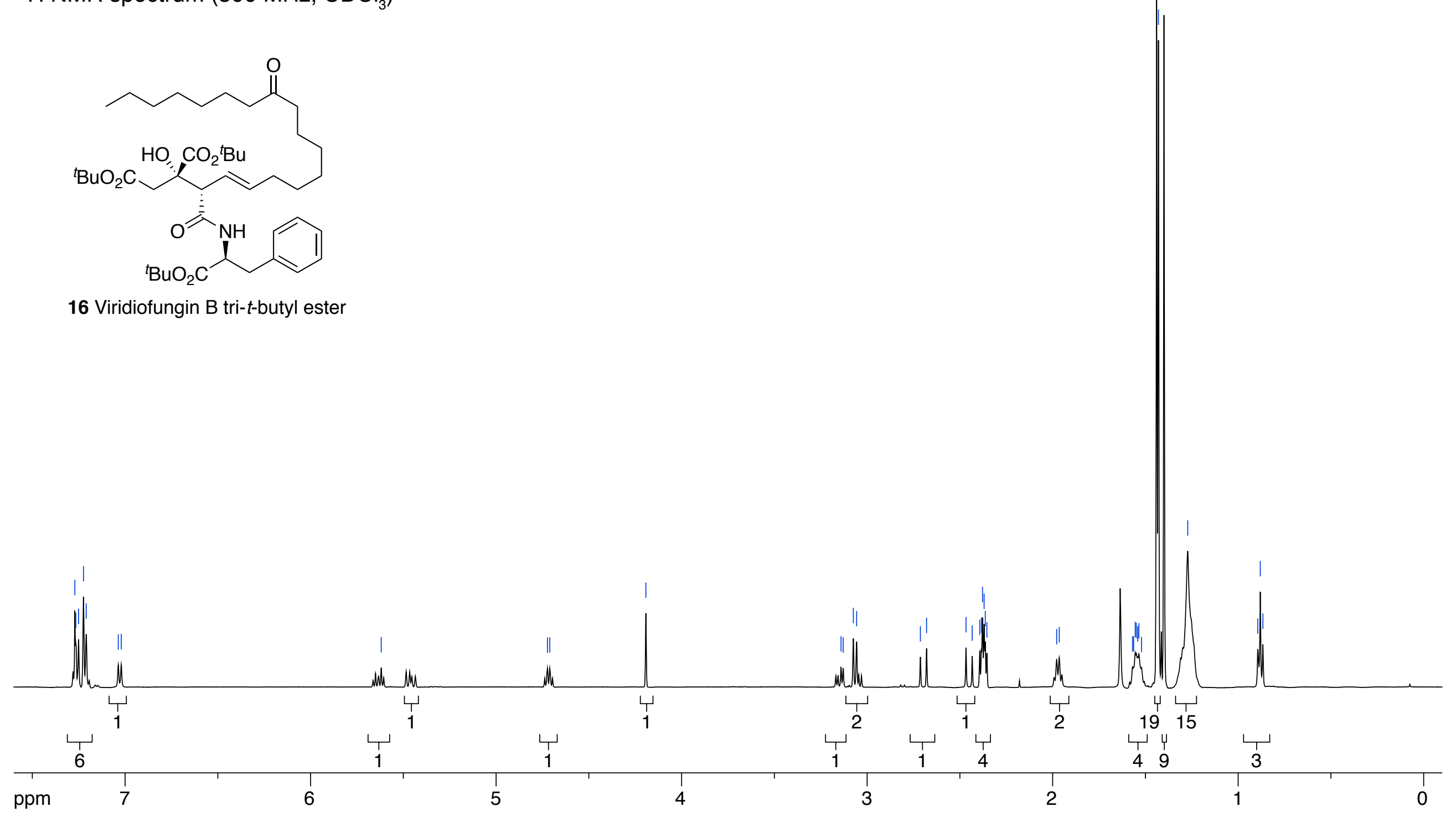

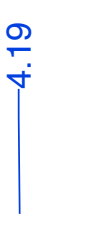

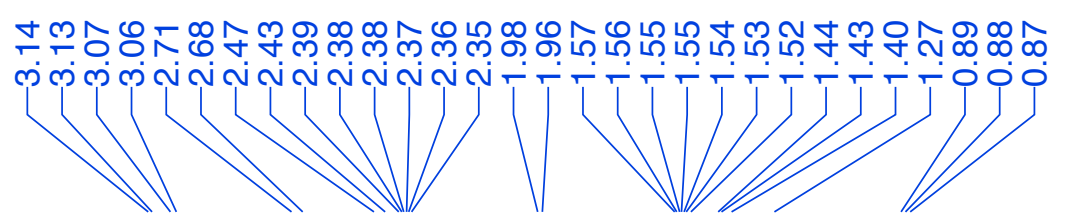




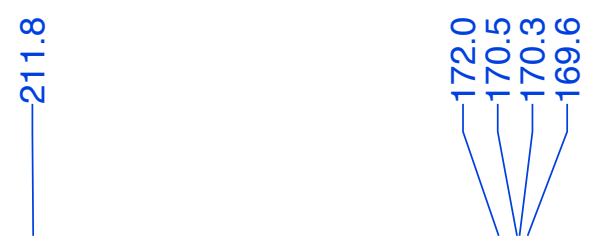

${ }^{13} \mathrm{C}$ NMR spectrum (125.5 MHz, $\mathrm{CDCl}_{3}$ )

16 Viridiofungin $B$ tri-t-butyl ester

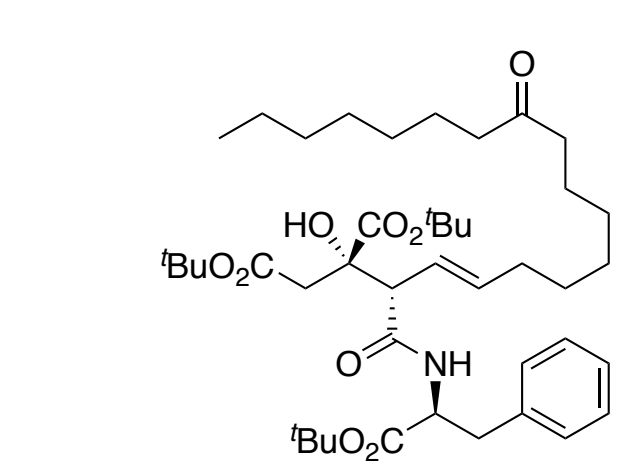

16 Viridiofungin B tritt-butyl ester

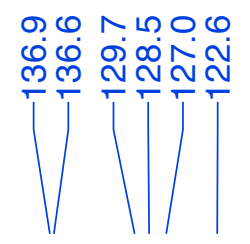

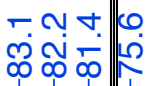

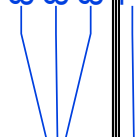

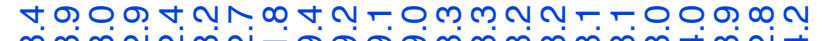

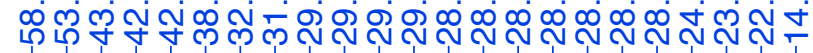

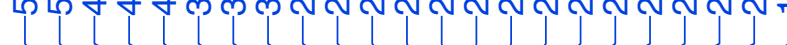

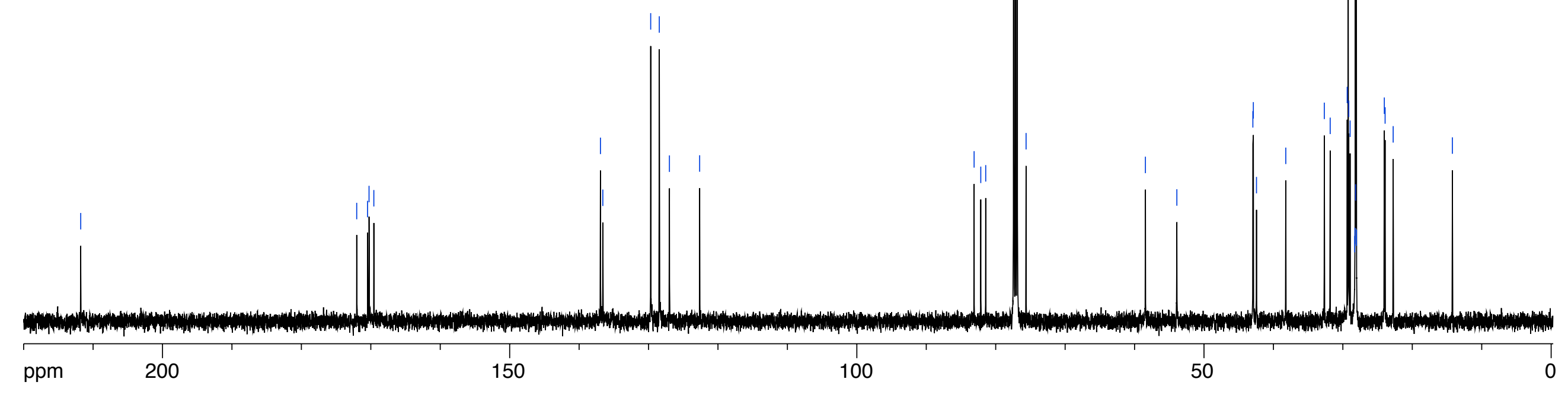




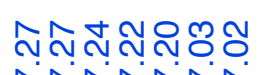

ペÑÑN

ใरी $\hat{\mid}\}$

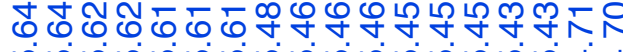

ம் ம்ட்ச்

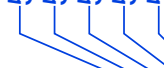

으

४

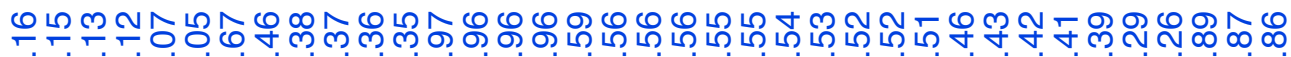

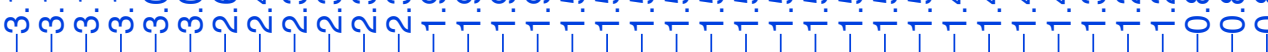

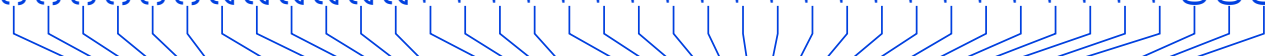

${ }^{13} \mathrm{C}$ NMR spectrum (125 MHz, $d_{4}$-methanol)

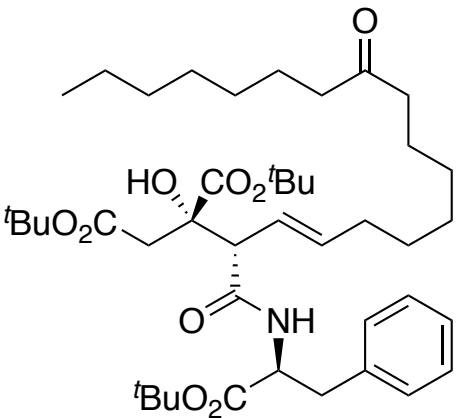

16 Viridiofungin $B$ tri-t-butyl ester
$+$

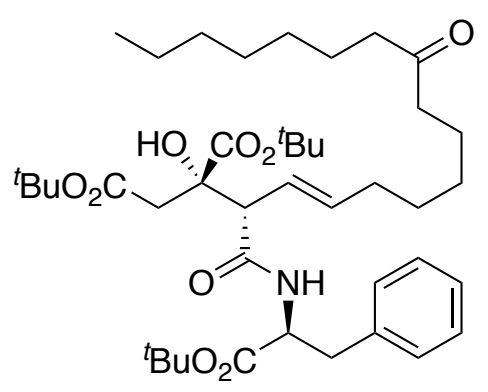

Desmethylene analogue tri-t-butyl ester

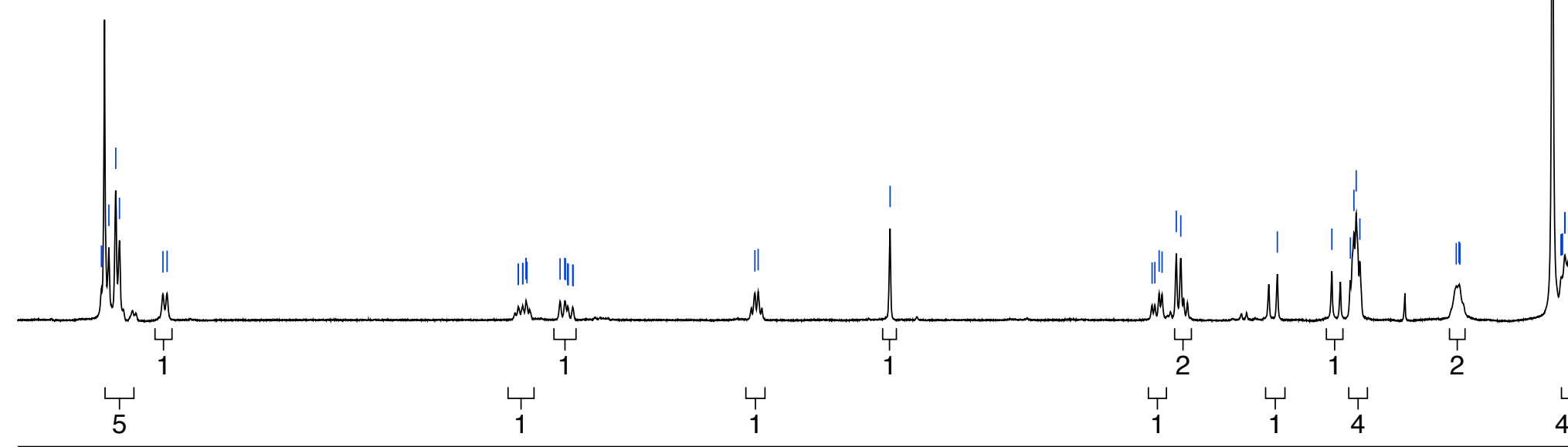




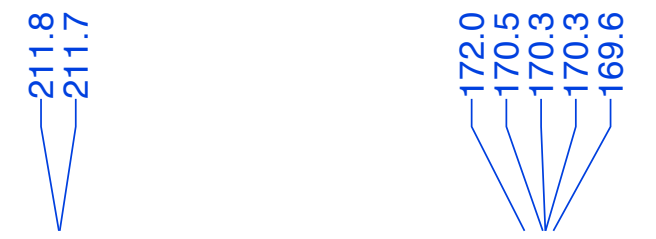

${ }^{13} \mathrm{C}$ NMR spectrum (125 MHz, $d_{4}$-methanol)

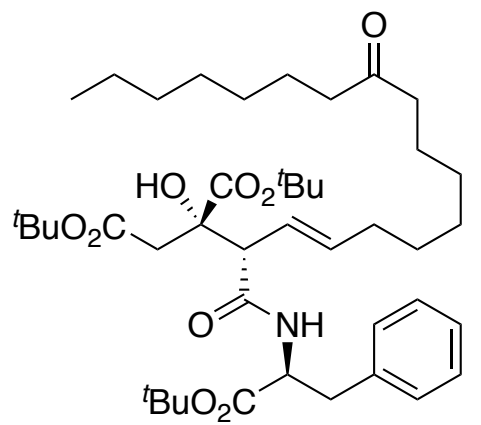

16 Viridiofungin $B$ tri-t-butyl ester
$0 \infty 0$ L mํำ

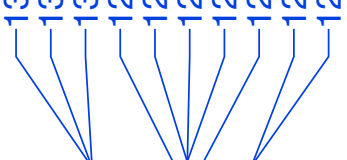

${ }^{t} \mathrm{CO}_{2} \mathrm{Hu}^{\mathrm{BO}}$

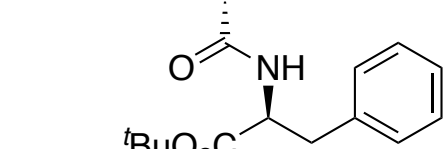

Desmethylene analogue tri-t-butyl ester

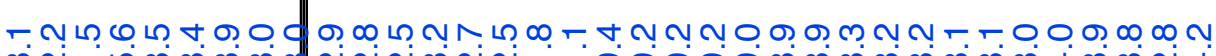
ळळळ

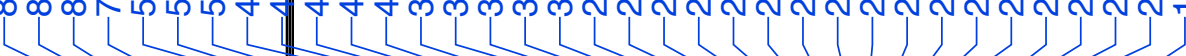

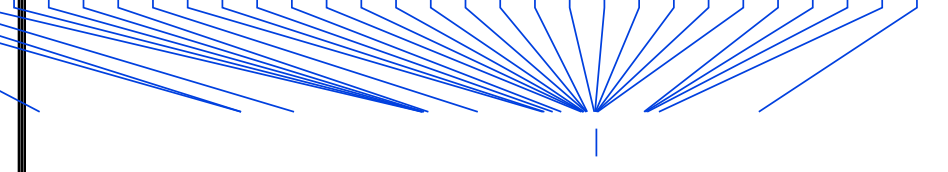

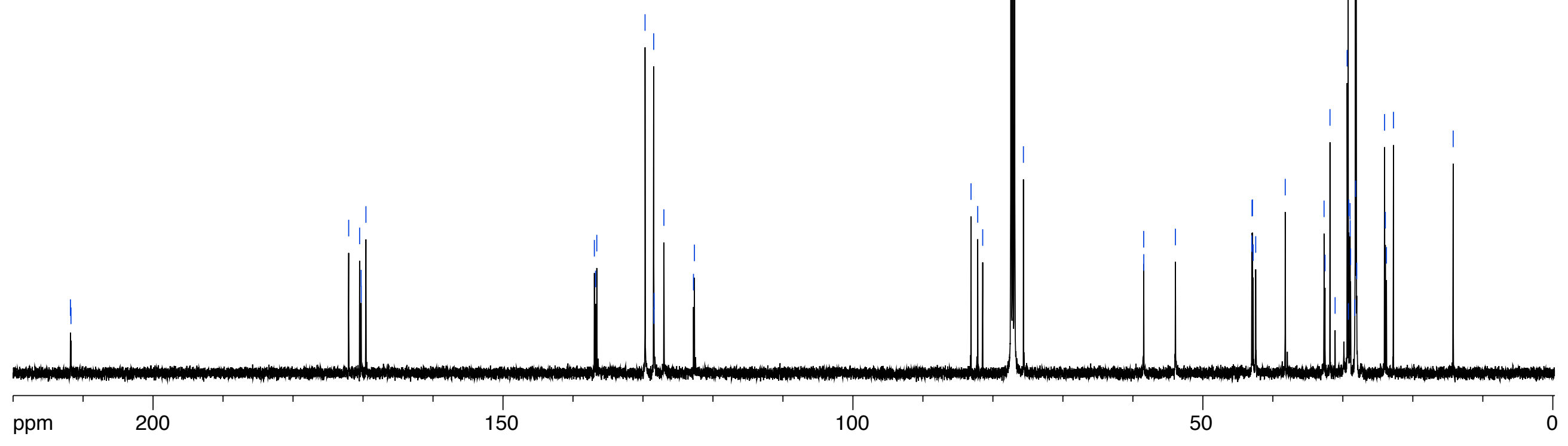




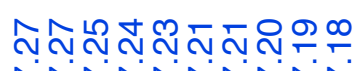

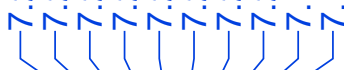

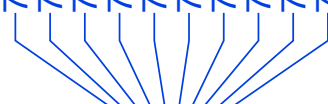

${ }^{1} \mathrm{H}$ NMR spectrum $\left(500 \mathrm{MHz}, d_{4}\right.$-methanol)

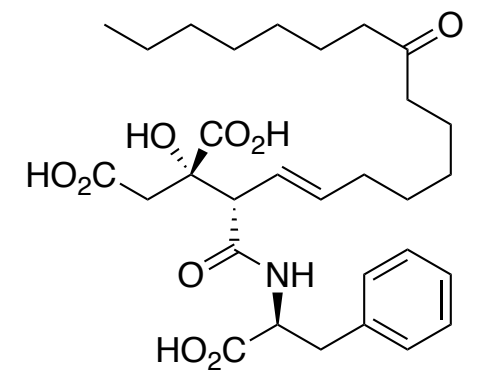

17 Desmethylene analogue

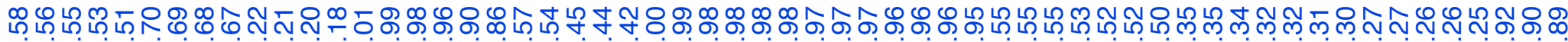
ம மமமம

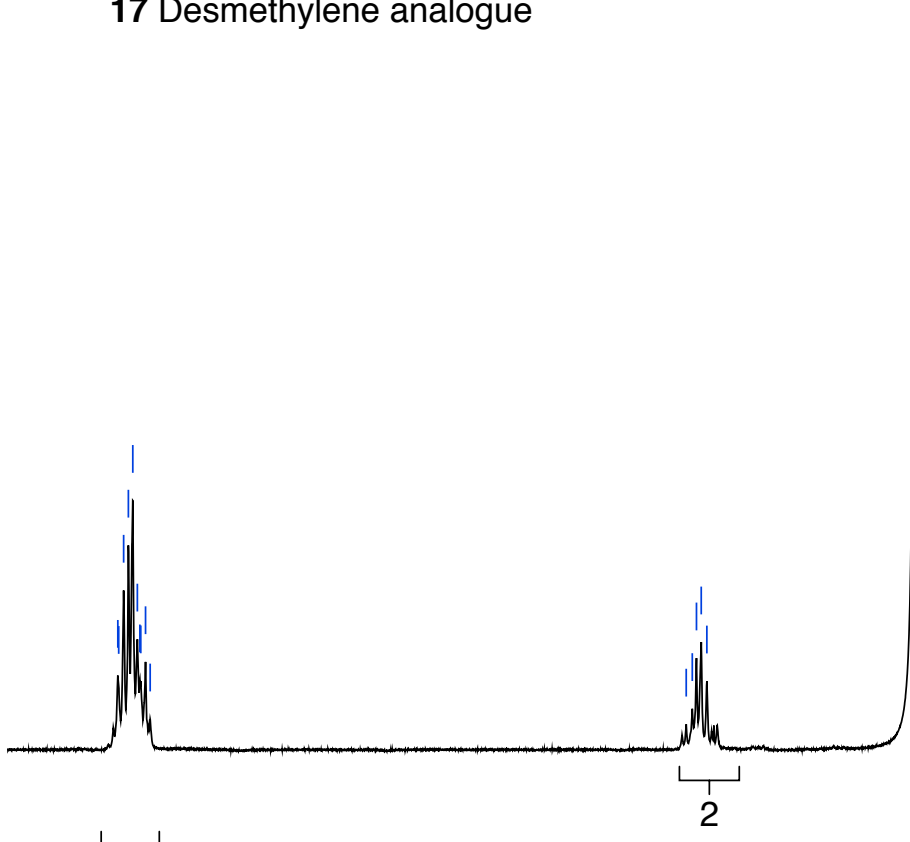

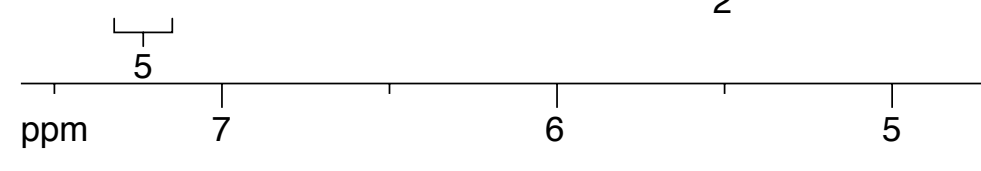
4
1

1

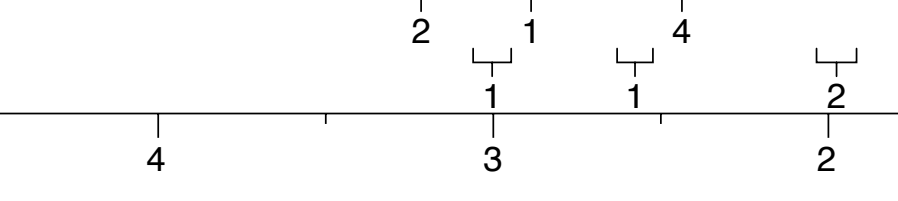

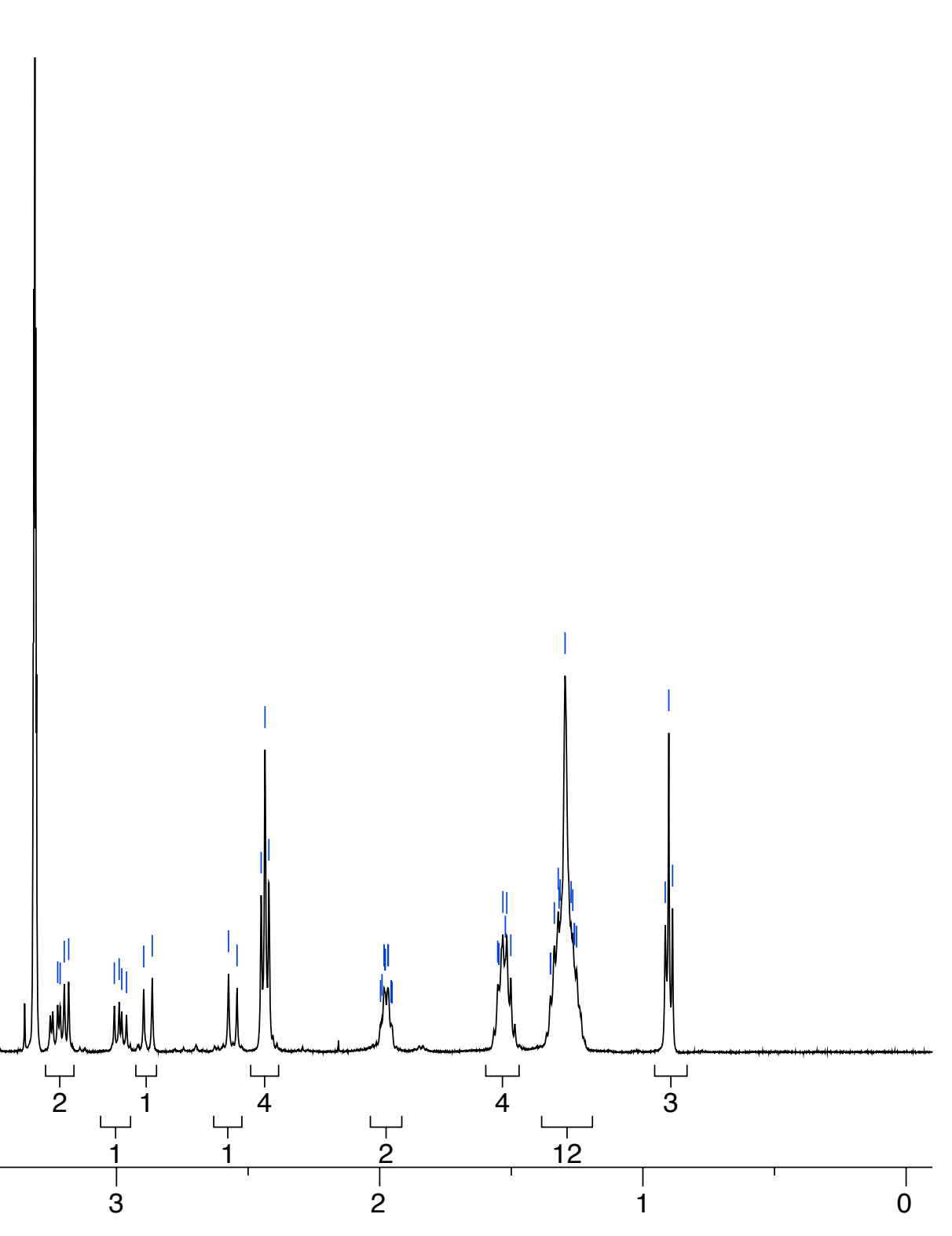



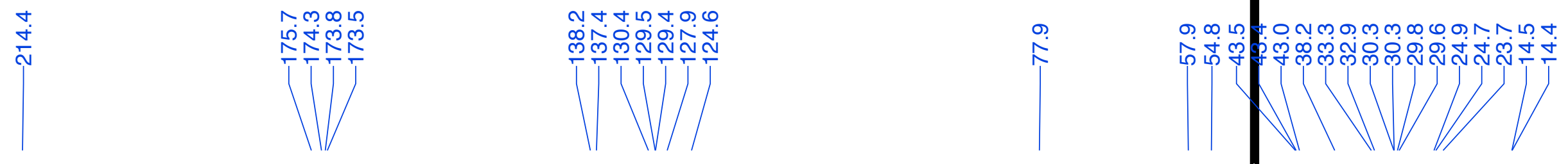

${ }^{13} \mathrm{C}$ NMR spectrum (125 MHz, $d_{4}$-methanol)

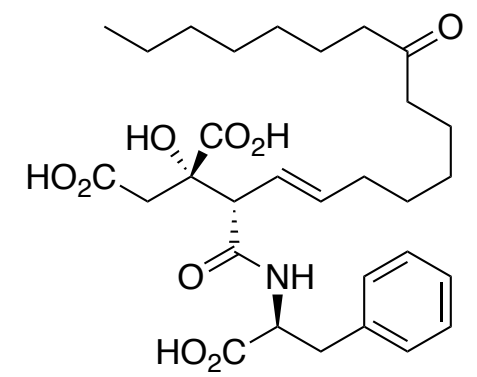

17 Desmethylene analogue

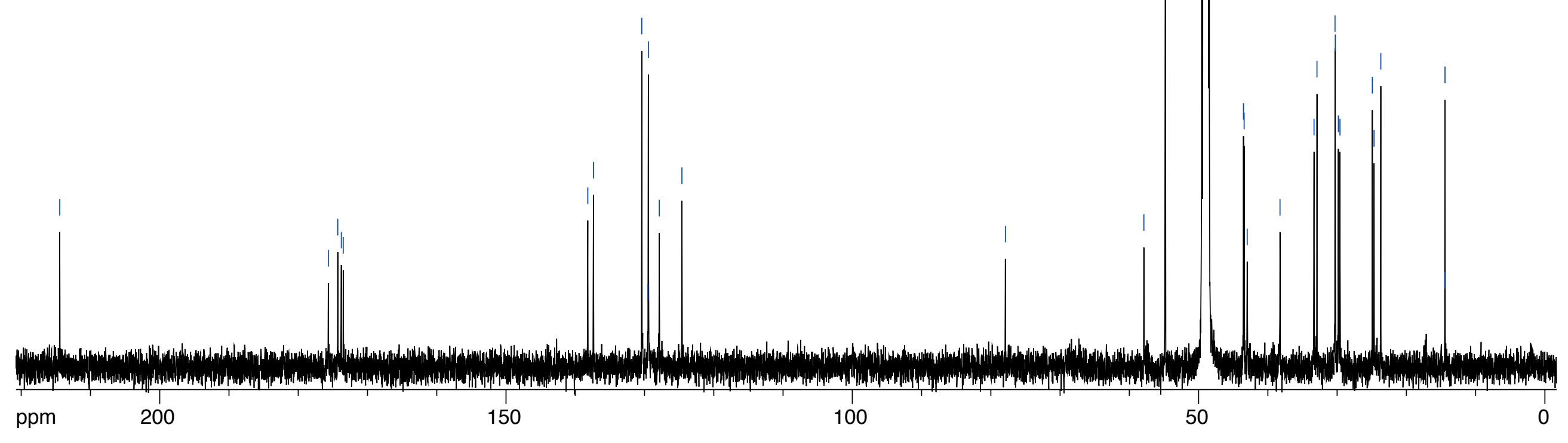




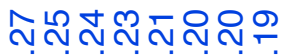

NヘNNNN

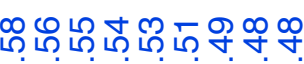
ए人乚

$8 \infty \infty 00$

+

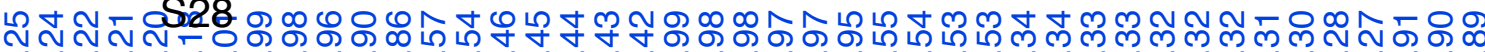

m m m m

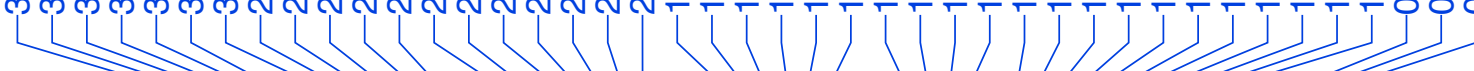

${ }^{1} \mathrm{H}$ NMR spectrum (500 MHz, $d_{4}$-methanol)

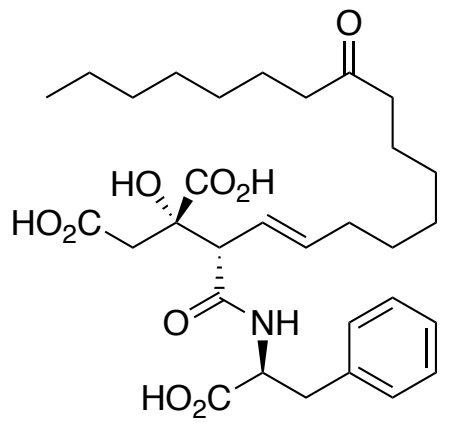

2 Viridiofungin $\mathrm{B}$
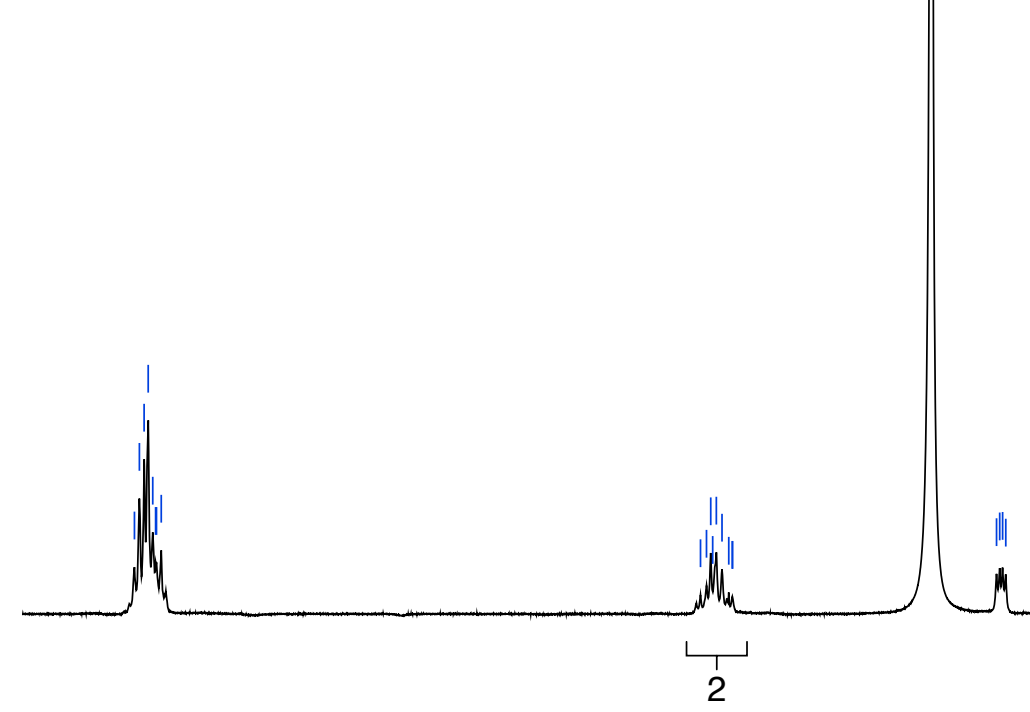

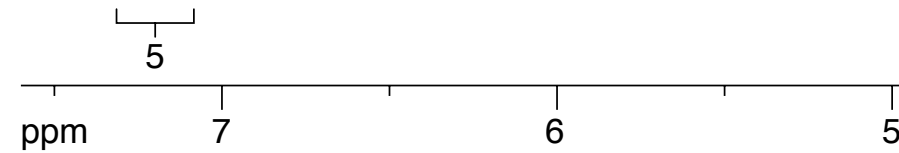

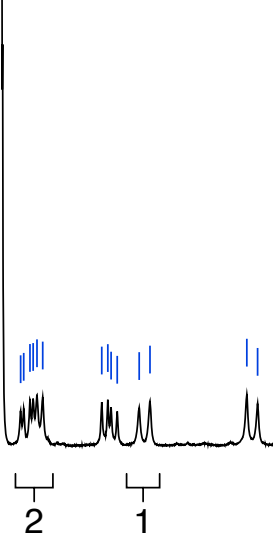

4
1
4 14 

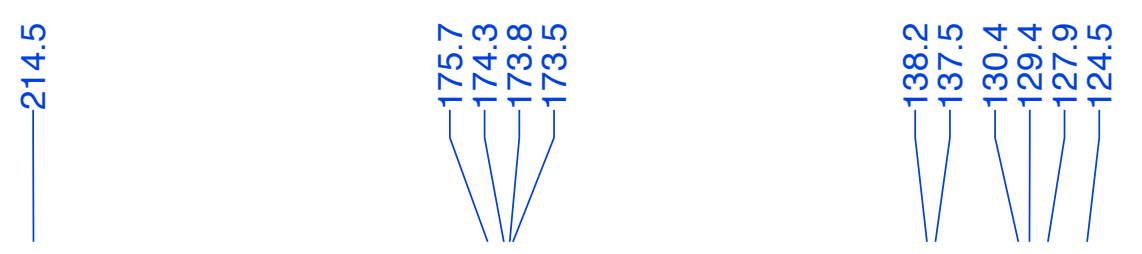

$\stackrel{\circ}{\wedge}$

๑ю

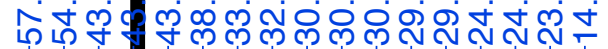

${ }^{13} \mathrm{C}$ NMR spectrum (125 MHz, $d_{4}$-methanol)

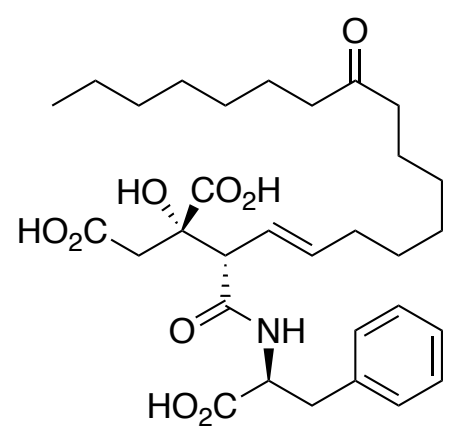

2 Viridiofungin $\mathrm{B}$

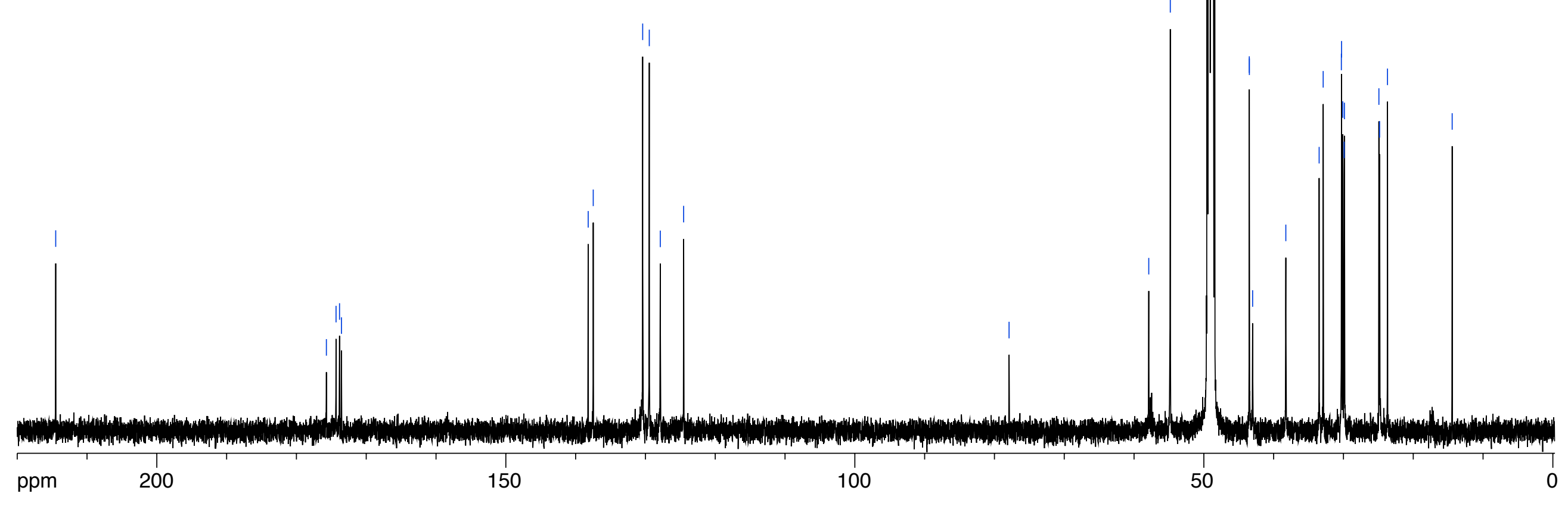


${ }^{13} \mathrm{C}$ NMR spectra (125 MHz, $d_{4}$-methanol)
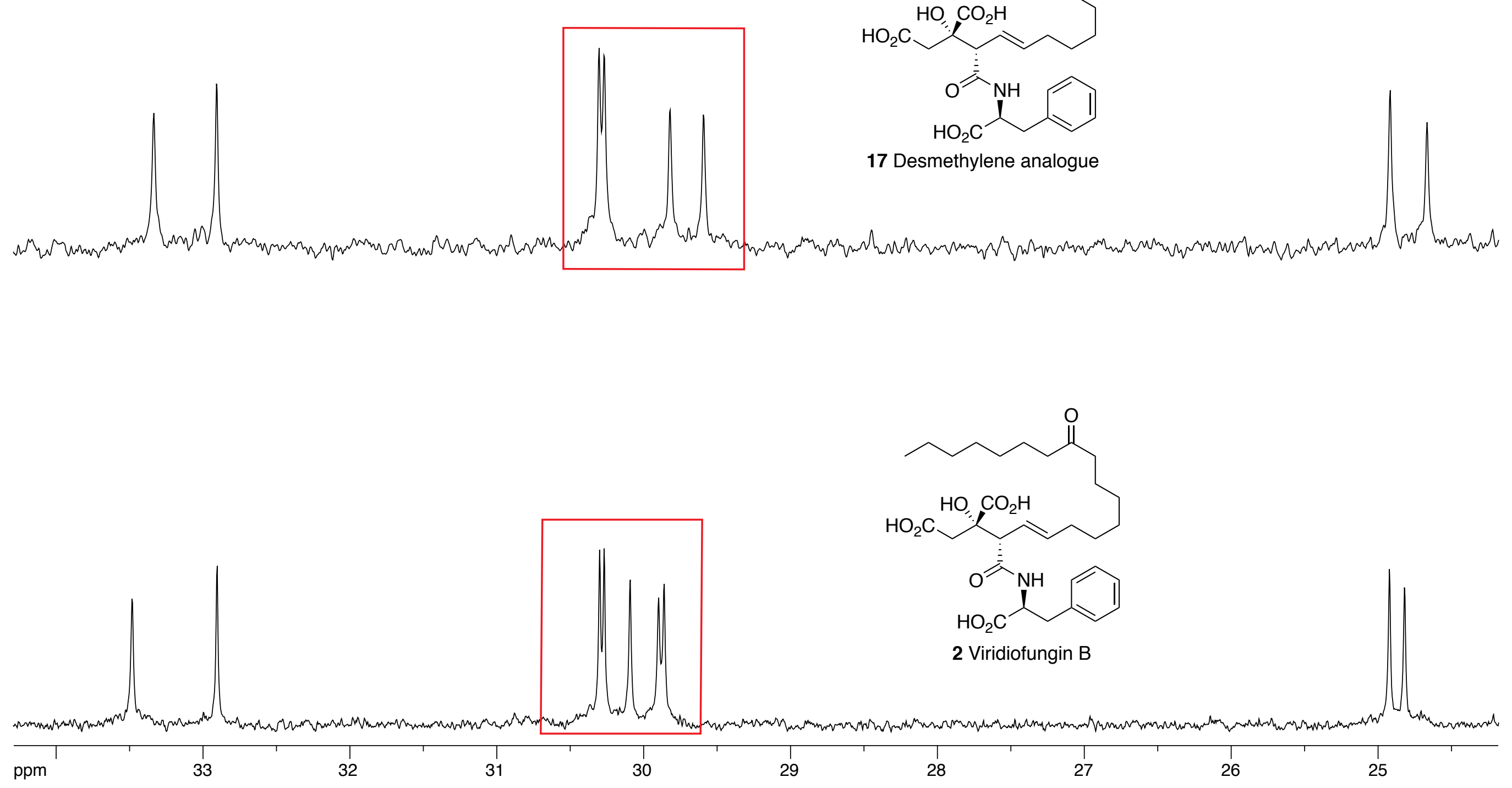

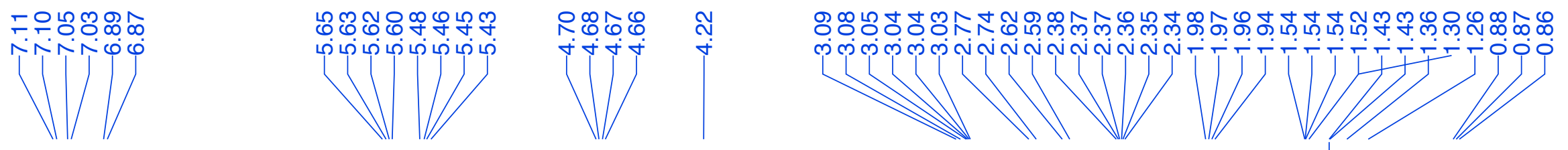

${ }^{1} \mathrm{H}$ NMR spectrum (500 MHz, $\mathrm{CDCl}_{3}$ )

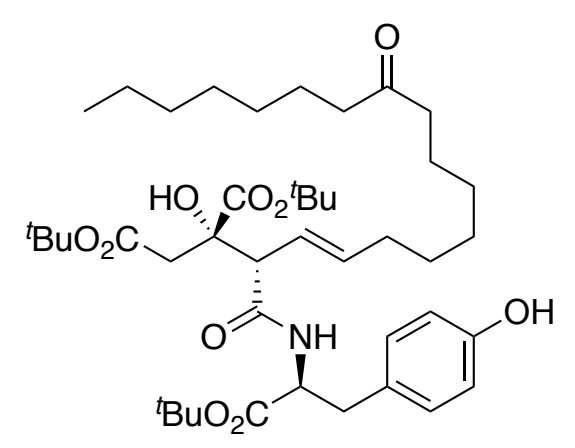

20 Viridiofungin A tri- $t$-butyl ester

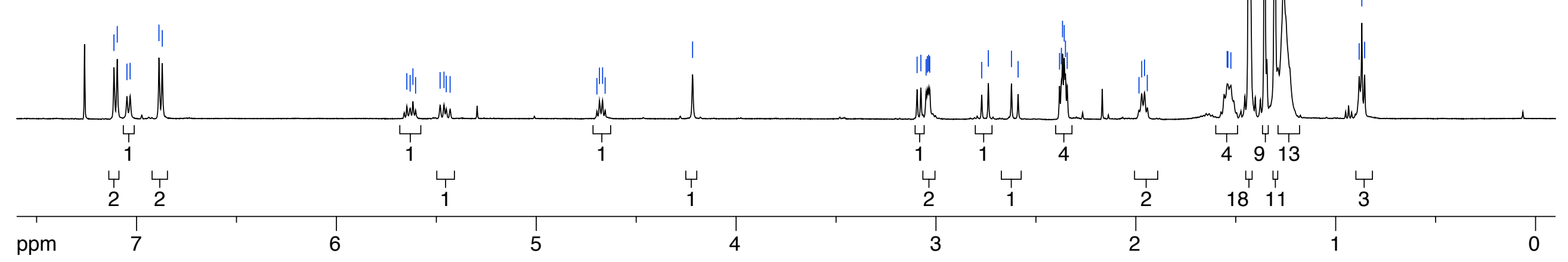




\section{S32}

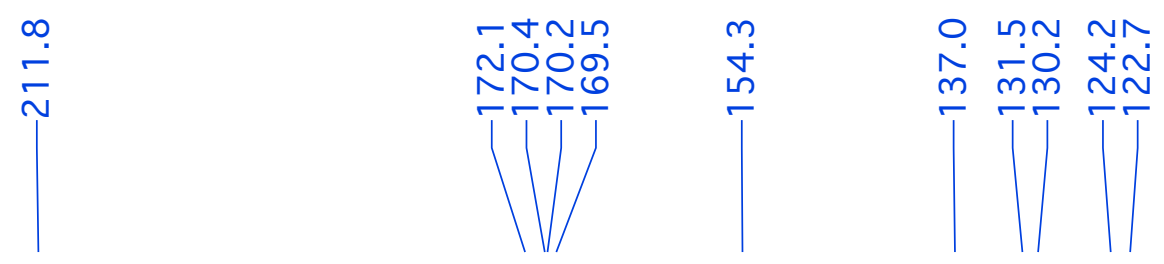

${ }^{13} \mathrm{C}$ NMR spectrum (125.5 $\mathrm{MHz} \mathrm{CDCl}_{3}$ )

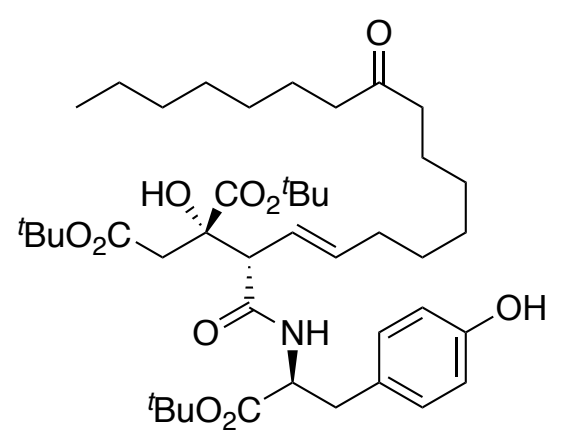

20 Viridiofungin A tri-t-butyl ester

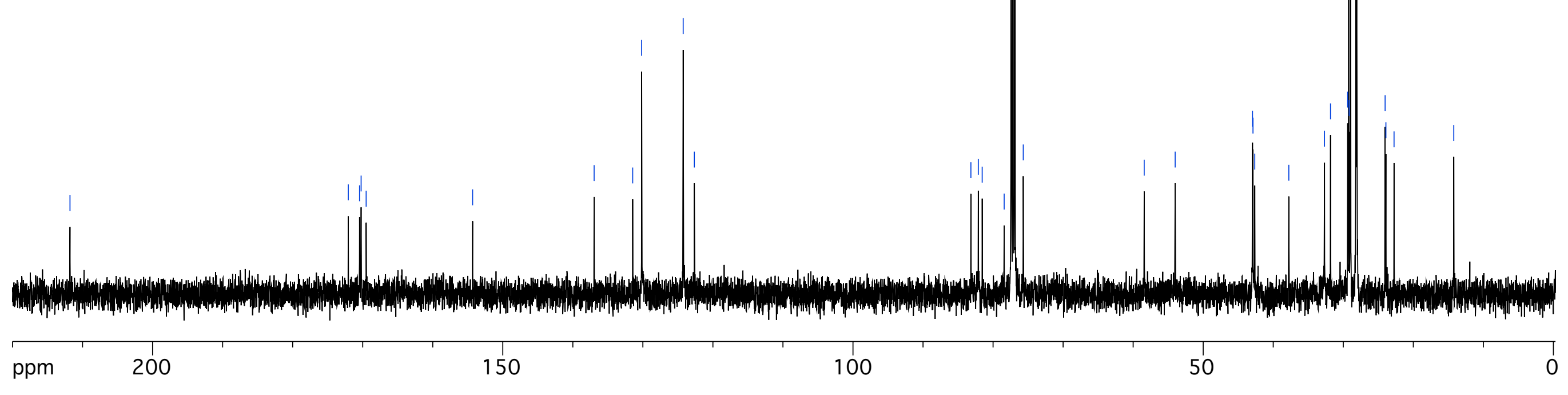




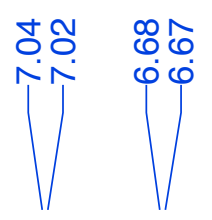

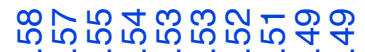

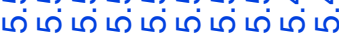

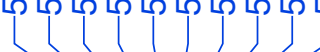

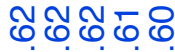

i t 4 A

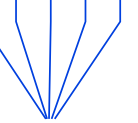

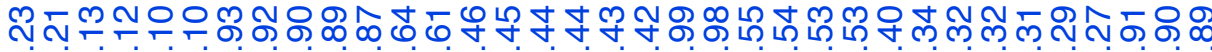

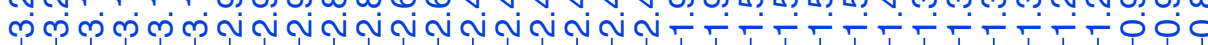

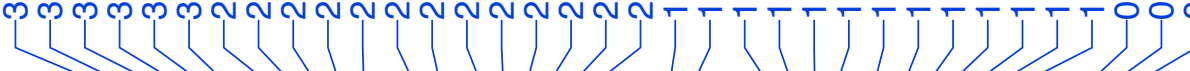

${ }^{1} \mathrm{H}$ NMR spectrum (500 MHz, $d_{4}$-methanol)
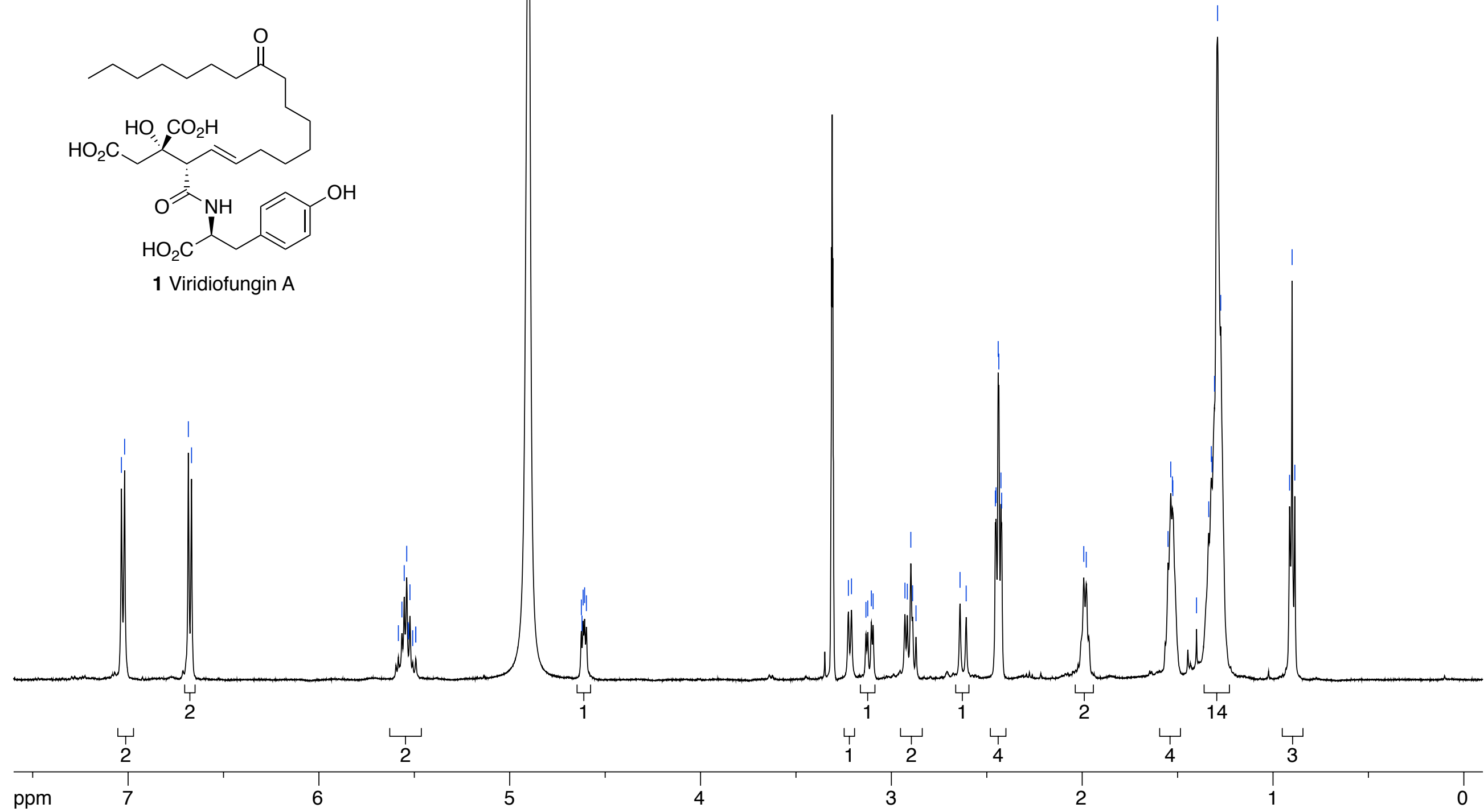


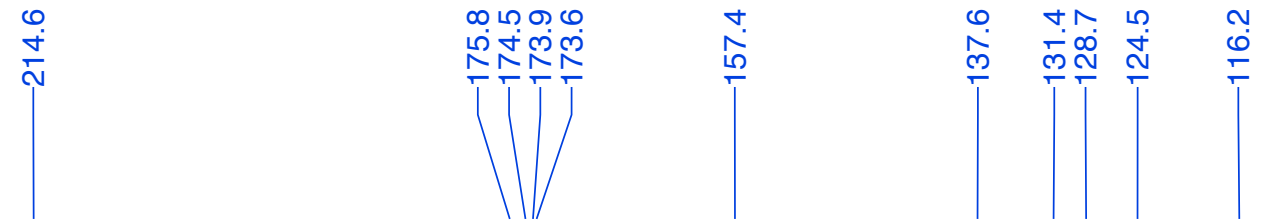

${ }^{13} \mathrm{C}$ NMR spectrum (125 MHz, $d_{4}$-methanol)

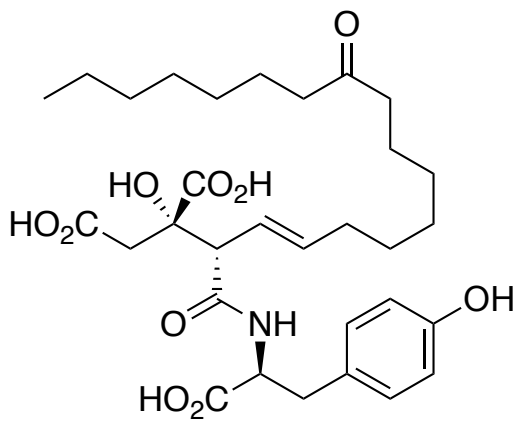

1 Viridiofungin A

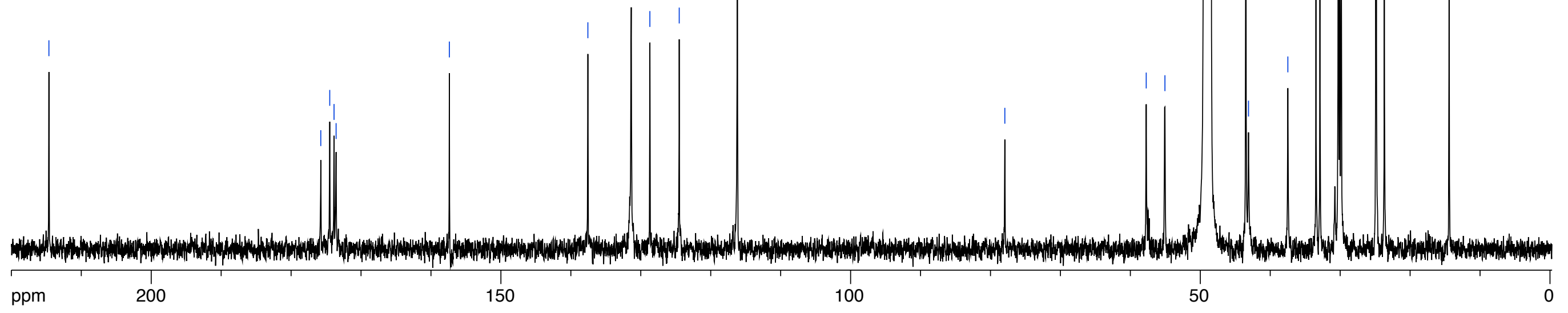

